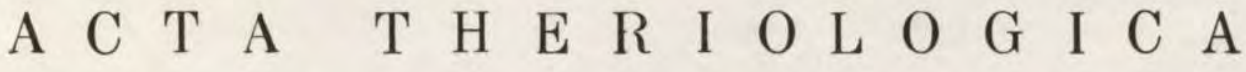 VOL. XII, 18: 293-322.

\section{Analysis einiger Parameter des Hundeschädels hinsichtlich ihres morphologischen Wertes ${ }^{1}$ )}

[Mit 12 Tabellen, 24 Abb. u. Tafeln XVI-XVIII]

\begin{abstract}
Untersuchungen über den morphologischen und taxonomischen Wert einiger Hundeschädelmerkmale (Canis lupus f. familiaris L in n a e us, 1758) wurden an 35 Schädeln erwachsener Hunde ( 8 weibliche und 27 männliche Exemplare) 19 verschiedener Rassen, deren Widerristhöhe von 20 bis $80 \mathrm{~cm}$ betrug, durchgeführt. Es wurden 52 Parameter betrachtet: A. 21 absolute Masse $(1-21)$, B. 20 ebensolche in Prozenten von $B-P$ ausgedrückt $(101,103-121), C$. 11 Quotienten-Indizes (201211). Eine Analysis dieser Merkmale ergab eine Einteilung dieser Parameter in genotypische und phenotypische, sowie auch in taxonomische und nicht-taxonomische. $\mathrm{Zu}$ den zur morphologischen Einschätzung des Hundeschädels am besten geeigneten Parametern (genotypisch-taxonomischen) gehören die folgenden: (A) von den absoluten Massen: $\mathrm{Or}-\mathrm{P}$, $N-P$, Sagekt-P, B-P, Backzahn-, Prämolar- und Molarreihenlänge, $O$-Sagekt, grösste Breite des Trigonum occipitale, $O-N$ und $B-E t h m$.; (B) von den in Prozenten des $B-P$ ausgedrückten Massen: $E u-E u$, Hirnschädelhöhe und eventuell noch $Z y-Z y$; (C) von den Quotienten-Indizes: Längen-Breiten-Index des Hirnschädels, Stirnlage-Index und Föhen-Längen-Index des Hirnschädels. Diese Merkmale unterscheiden die in Gestalt und Grösse verschiedenen Hundeschädel am meisten. Die Variabilität betreffend, scheinen die Schädel mehr in Grösse als in Gestalt differenziert zu sein. Den stabilsten Schädelteil bildet der Hirnschädel, den labilsten - das Stirndreieck (seine Höhe) und die Stirn in dem grössten (Ekt-Ekt) und kleinsten (Ent-Ent) Breitenmass.
\end{abstract}

\section{EINLEITUNG}

Allgemein ist bekannt, dass der Hund (Canis lupus forma familiaris L in n a e us, $1758)^{2}$ ) von allen Haustieren die grösste Variabilität der Gestalt und Grösse aufweist. Die Differenzen zwischen zwei Hunden in dieser Hinsicht, besonders aber

1) Vorgetragen auf dem Jubiläumskongress der polnischen Morphologen, Gdańsk, 20.-23.IX.1964

2) Eine neue Nomenklatur (Bohlken, 1961), die sich auf die monophiletische Abstammung des Hundes beruft. 
hinsichtlich der Gestalt des Kopfes, können so gross sein, dass man sie nicht nur auf Rassen- sondern sogar auf Gattunsunterschiede beziehen könnte, wäre die Gattung nur durch diese Parameter charakterisiert. Weil dies jedoch nicht so ist, entsteht ein für den Morphologen sehr interessantes Problem des Aussehens des Skeletts, im besonderen des Schädels dieser Tiere. Es können die folgenden Fragen gestellt werden: Welche von den Schädelmerkmalen unterscheiden die Hunde am besten, wenn man von Geschlecht und Rasse absieht? Welche Parameter können vom morphologischen Standpunkt den Schädel dieses Tieres am günstigsten und bequemsten charakterisieren?

Wie aus dem zahlreichen Schrifttum hervorgeht, wurde seit langher und auf verschiedene Art und Weise versucht dieses Problem zu lösen; genannt seien hier die Beiträge von St u der (1901), K lat t (1913), Brinkman n (1921,1924), S c h äme (1922), Götze \& Dornheim (1926), M a r chlewski (1926, 1930), Bogo1 ubskij (1928), Wagner (1929) und Duerst (1930), deren Autoren entweder auf zeitgenössischen oder archäologischen Material basierten. Die erwähnten Unterschiede zwischen einzelnen Individuen versuchte man teils durch Untersuchung von absoluten Massen, teils mittels relativer Masse (in Prozenten der Basallänge ausgedrückt), die die Gestalt erfassen solten, und schliesslich durch Quotienten-Indizes verschiedener absoluter Masse auszudrücken. Eine genaue Statistik würde etwa 80 absolute Parameter, ebensoviele relative Parameter und eine gewisse Zah. von Indizes des dritten Typs im Schrifttum auffindbar machen.

Von den absoluten Massen wird mit der Basallänge (Basion-Prosthion) und mit der inneren Hirnhöhlenlänge (Basion-Ethmoideum), die nach K lat t (1913) bei Individuen der gleichen Grösse sehr stabil ist und sich deshalb ale idealer Parameter für die Grösse des Tieres eignet, sowie mit einigen Kiefermassen, die nach B r in k$\mathrm{m}$ a n n (1924) und $\mathrm{Dahr}$ (1937) ebenfalls zur Berechnung der Basallänge des Schädels dienen können, ein grosser morphologischer Wert verbunden. Von den relativen Massen möchte man in dieser Beziehung den Stirnlage-Index von Brinkmann (1921), welcher sich gut zur Differenzierung der kurz- und langschnauzigen Schädel eignet, und den Breiten-Längen-Index des Hirnschädels (W a g n e r, 1929), der mit der Tiergrösse negativ korreliert ist, nennen. Eine gewisse Bedeutung für die morphologische Beurteilung des Hundeschädels sollen auch nach G eorg i (1937) gewisse am Kiefer gemessene Winkel und nach W a gner (1929) der Radius der Gesichtskrümmung haben.

Die Zahl der in dieser Hinsicht gewonnenen Beobachtungen ist demnach ziemlich gross. Dennoch ist bisher sehr wenig über den mórphologischen und taxonomischen Wert dieser Merkmale und uber ihre gegenseitigen Abhängigkeiten bekannt, Das Ziel dieses Beitrages ist, hier eine kleine Lücke zu füllen.

\section{UNTERSUCHUNGSMATERIAL UND METHODIK}

Die von uns begonnenen Untersuchungen wurden auf einem nicht sehr zahlreichen, aber dafür in Grösse und Gestalt sehr unterschiedlichen Material, welches aus Schädeln (Calvaria) von 35 erwachsenen Hunden 19 verschiedener Rassen mit Widerristhöhe von 20 bis $80 \mathrm{~cm}$ bestand, geführt. Insgesamt haben wir 27 Schädel von männlichen und 8 Schädel von weiblichen Exemplaren untersucht (Tabelle 1). Diese Schädel wurden uns von Rassenhunden-Züchtern des Bezirks Wrocław und von Frau Veterinärarzt Danuta Hryniewicz von der Rassenhundezucht „Korde- 
Tabelle 1.

Charakteristik des Untersuchungsmaterials.

\begin{tabular}{|c|c|c|c|c|c|c|}
\hline $\begin{array}{l}\text { Ifed. } \\
\text { Nr. }\end{array}$ & Rasse & Inw. - Nr. & $\begin{array}{l}\text { Geschlecht } \\
\text { I oder W }\end{array}$ & $\begin{array}{c}\text { Widerrist- } \\
\text { höhe in cm } \\
\text { Iw/ }\end{array}$ & \multicolumn{2}{|c|}{$\begin{array}{l}\text { Inn. H1rn- } \\
\text { höhlenlge. } \\
\text { in mm }\end{array}$} \\
\hline 1 & Ratle Plnscher & $56 / 1$ & * & 20,0 & 52,0 & \\
\hline 2 & Pekinese & $86 / 32$ & is & 26,0 & 55,0 & \\
\hline 3 & Grossspltz & $55 / 3$ & u & 38,5 & $66,0\}$ & 67.0 \\
\hline 4 & Grossspitz & $47 / 2$ & $w$ & 38,0 & $68,0\}$ & 67,0 \\
\hline 5 & Kurzhaar-Dachshund & $64 / 5$ & M & - & 69,0 & \\
\hline 6 & Skye-Terrier & $63 / 6$ & w & 31,0 & 70,0 & \\
\hline 7 & Poln1scher THefland-Schäferh. & $85 / 33$ & y & 52,0 & $78,5\}$ & 78,8 \\
\hline 8 & Poln1scher Te1fland-Schäferh. & $74 / 4$ & M & 48,0 & $79,0\}$ & 78,8 \\
\hline 9 & Bulldogge & 13 & u & - & 79,0 & \\
\hline 10 & Boxer & $84 / 34$ & M & 56,0 & 78,07 & \\
\hline 11 & Boxer & $81 / 15$ & M & 60,0 & $87,0\}$ & 84,3 \\
\hline 12 & Boxer & $29 / 14$ & M & 61,0 & 88,0 & \\
\hline 13 & Deutsch Drahthaar & $7 / 9$ & is & - & 86,0 & \\
\hline 14 & Deutsch Kurzhaar & $73 / 10$ & if & 56,0 & 87,0 & \\
\hline 15 & Ir1sh Setter & $53 / 11$ & M & 58,0 & $86,0\}$ & 89,5 \\
\hline 16 & Ir1sh Setter & $80 / 12$ & $u$ & 63,0 & $93,0\}$ & 89,3 \\
\hline 17 & Afredale-Terrie: & $5 / 7$ & " & 55,0 & 91,0 & \\
\hline 18 & Span1el /?/ & $54 / 8$ & M & 57,5 & 92,0 & \\
\hline 19 & Deutscher Schäferhund & $6 / 25$ & u & 58,0 & $93,0)$ & \\
\hline 20 & Deutscher Schäferhund & $16 / 26$ & M & 64,0 & $95,0\}$ & 93,6 \\
\hline 21 & Deutscher Schäferiunã & $83 / 35$ & x & 68,0 & $96,0)$ & \\
\hline 22 & Coll1e & $67 / 27$ & w & 62,0 & 90,0 & \\
\hline 23 & Collie & $61 / 28$ & M & 71,0 & $96,0\}$ & 94,0 \\
\hline 24 & Coll1e & $49 / 29$ & M & - & $96,0)$ & \\
\hline 25 & Poln1scher Bergland Schäferh. & $82 / 24$ & w & - & 95,0 & \\
\hline 26 & Bernhard1ner & $76 / 19$ & M & 67,0 & $92,0)$ & \\
\hline 27 & Bernhardiner & $71 / 21$ & M & - & 96,0 & \\
\hline 28 & Bernhard1ner & $13 / 20$ & M & 75,0 & 100,0 & 99,6 \\
\hline 29 & Bernhard1ner & $33 / 22$ & M & 80,0 & 104,0 & \\
\hline 30 & Bernhard1ner & $72 / 23$ & us & 69,5 & 106,0 & \\
\hline 31 & Deutsine Dogge & $69 / 16$ & $w$ & - & 99,0 & \\
\hline 32 & Deutsche Dogge & $62 / 18$ & M & - & 102,0 & 102,0 \\
\hline 33 & Deutsche Dogge & $21 / 17$ & M & 75,0 & 105,0 & \\
\hline 34 & Barsn1 & 30 & u & - & 103,0 & 104,5 \\
\hline 35 & Barso: & 31 & M & - & $106,0\}$ & \\
\hline
\end{tabular}

garda" in Leba ${ }^{3}$ ) zu Verfügung gestellt. An jedem von diesen Schädeln wurden 21 Merkmale (Brinkmann, 1921; Duerst, 1930; Wagner, 1929; B ibikova, 1953) gemessen (Abb. 1), und zwar die folgenden:

a) Längenmasse

1. Basion-Ethmoideum (B-Ethm.)

2. Basion-Prosthion $(B-P)$

3. Opisthion-Nasion $(O-N)$

4. Nasion-Prosthion $(N-P)$

5. Opisthion-Sagektorbion $\left.{ }^{4}\right)(\mathrm{O}-$ Sagekt $)$

3) Allen Spendern möchten wir hier unseren aufrichtigen Dank aussprechen.

4) Sagektorbion $=$ Stirnmitte, d.h. Schneidepunkt der Linien, welche die Enden der Jochfortsätze des Frontale mit der Mittelebene des Schädels verbinden (R e$m$ é n y i, 1954). 
6. Sagektorbion- Prosthion (Sagekt-P)

7. Höhe (Länge) des Trigonum frontale, vom Sagektorbion zum Treffpunkt der beiden Crista frontalis ext. mit den Crista sagittalis ext. gemessen. Im Falle, dass sich die Crista frontalis ext. auf dem Schädel nicht treffen, wird dieses Mass vom Sagektorbion bis zum Treffpunkt der Verlängerungen der beiden Crista frontalis

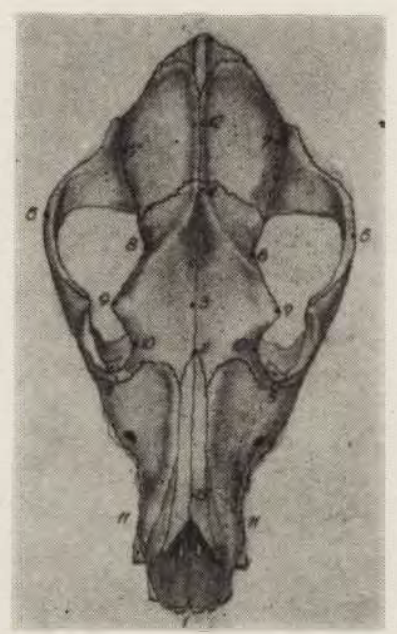

Abb. 1. Die wichtigsten Messpunkte des Hundeschädels in der Stirnebene: 1 - Prosthion $(P), 2$ - Nasion $(N), 3$ - Sagektorbion (Sagekt), 4 - Apex trigoni front., 5 Orbitale (Or), 6 - Zygion $(\mathrm{Z} y), 7$ - Euryon $(E u)$, 8 - Frontcstenion $(F r), 9$ - Ektorbitale (Ekt), 10 - Entorbitale (Ent), (11-11: kleinste Gaumenbreite), 12 - Fornix neurocranii, 13 - Fornix nasi.

ext. ausserhalb des Schädels gemessen (gemessen wird auf einem Papierblatt, auf welchem der entsprechende Schädelteil vorher aufgezeichnet wurde).

8. Orbitale-Prosthion $(\mathrm{Or}-\mathrm{P})$

b) Breitenmasse

9. Zygion-Zygion $(Z y-Z y)$

10. Grösste Breite des Trigonum occipitale

11. Euryon-Euryon $(E u-E u)$

12. Frontostenion-Frontostenion $(F t-F t)$

13. Ektorbitale-Ektorbitale (Ekt-Ekt)

14. Entorbitale-Entorbitale (Ent-Ent)

15. Kleinste Gaumenbreite auf der Höhe der Prämolaren gemessen.

c) Höhenmasse

16. Hirnschädelhöhe: vom Sphenobasion lotrecht aufwärts zum Fornix neurocranii ohne den Crista sagittalis ext.

17. Nasalhöhe: von der Verbindungslinie der Proc, alveolores der Ossa maxillaria zwischen $P_{1}$ und $P_{2}$ lotrecht aufwärts zum Fornix nasi.

d) Zahnmasse

18. Länge der Backzahnreihe $(M+P)$ : Kronenmass

19. Länge der Molarreihe $(M)$ : Kronenmass

20. Länge der Prämolarreihe $(P)$ : Kronenmass

21. Länge des Reisszahnes $\left(P_{4}\right)$, an der Aussenseite gemessen. 
Die eben aufgezählten Merkmale wurden nachher auch (ausser Nr. 2) in Prozenten der Basallänge ausgedrückt (sie erhielten die Bezeichnungen 101, 103-121) und von einigen von ihnen wurden die folgenden elf Quotienten-Indizes aufgestellt: 201. Längen-Breiten-Index des Hirnschädels: $(O-$ Sagekt/Eu-Eu $) \times 100$

202. Stirndreieck-Index: (Höhe des Trigonum frontale/Ekt $-E k t) \times 100$

203. Stirnbreiten-Index: $(E k t-E k t / B-P) \times 100$

204. Höhendifferenz-Index: (Hirnschädelhöhe/Nasalhöhe) $\times 100$

205. Stirnlage-Index: (Sagekt $-P / O-$ Sagekt $) \times 100$

206. Freitendifferenz-Index: $(Z y-Z y /$ kleinste Gaumenbreite $) \times 1 c 0$

207. Höhen-Längen-Index des Hirnschädels: (Hirnschädelhöhe/B-Ethm.) $\times 100$

208. Ausprägungs-Index des Hirnschädels: $(E u-E u / F t-F t) \times 100$

209. Breiten-Höhen-Index des Hirnschädels: (Eu-Eu/Hirnschädelhöhe) $\times 100$

210. Backzahn-Index: (Molarenlänge + Prämolarenlänge/Backzahnlänge) $\times 100$

211. Reisszahn-Index: (Reisszahnlänge/Molarenlänge) $\times 100$

Insgesamt haben wir demnach 52 Parameter betrachtet: 21 absolute Masse, die die Nummern 1-21 tragen und weiterhin Masse (oder Parameter) der Gruppe A genannt werden, 20 absolute Masse in Prozenten der Basallänge ausgedrückt, die (iie Nummern 101 und 103-121 tragen und weiterhin Masse der Gruppe B genannt werden, und 11 Quotienten-Indizes, die die Nummern 201-211 tragen und Parameter der Gruppe C weiterhin genannt werden.

Der morphologische und taxonomische Wert dieser Parameter wurde durch Untersuchung von Korrelationen und Variationskoeffizienten, durch Vergleich der erhaltenen Werte mit denen der Schädelparameter des Wolfes iCanis lupus Linn a eus, 1758) und durch Einteilung des Materials in vorgeschlagene Grössenklassen beurteilt. Ausserdem haben wir Czekanowski-Tafeln und Graphen (Wrocław-Taxonomie-Methode; Perkal, 1953) aufgestellt, wobei die Entfernung der Individuen $x$ und $y$ bei Berücksichtigung von $n$ Merkmalen nach der folgenden Formel berechnet wurde:

$$
d=\sqrt{\frac{1}{n} \sum_{1}^{n}\left(x_{i}-y_{i}\right)^{2}}
$$

Für jeden dieser Parameter haben wir auch den Variationsbereich $Z$, den Mittelwert $\bar{x}$ und den Standardfehler $S$ (P e r k a 1, 1958-1963) berechnet. Als hoch korrelierte Parameter werden wir weiterhin solche bezeichnen, deren Korrelationkoeffizient $r$ den Wert von $90 \%$ überschreitet $(r \geqslant 0,9)$. Alle Berechnungen wurden im Fiechenzentrum der Universität Wrocław auf dem Rechenautomaten Elliott-803 ausgeführt $\left.{ }^{5}\right)$.

\section{ANALYSIS DER PARAMETER}

\section{A. Absolute Masse}

Wie schon erwähnt, wurden 21 Merkmale dieser Gruppe analysiert: \& Längenmasse (Nr. 1-8), 7 Breitenmasse (Nr. 9-15), 2 Höhenmasse (Nr. 16, 17) und 4 Zahnmasse (Nr. 18-21) (Tabelle 2).

5) Wir danken herzlichst Herrn Dozent Dr. S. Pas zkowski, dem Leiter des Instituts für Numerische Mathematik, welchem das Rechenzentrum angegliedert ist, für das grosszügige Vorsehen von Rechenzeit. 
a) Die Längenmasse. Wie aus den berechneten Korrelationen (Tabellen 3 und 4) und aus der in der Tabelle 2 enthalten Charakteristik hervorgeht, sind alle Längenmasse (ausgenommen die Höhe des Trigonum frontale) untereinander und mit dem Längen-Breiten-Index des Hirnschädels ( $\mathrm{Nr}$. 201) hoch korreliert. Ausserdem weist $B-E t h m$. eine grosse Abhängigkeit von der Grösse des Tieres $(r=96,1 \%$ ), der Widerristhöhe (W y r o s t $\& \mathrm{~K} \mathrm{u}$ ch a r c z y k, 1967a) auf, was auf einen bedeutenden taxonomischen Wert dieses Parameters und dadurch auch in diesem Sinn auf die mit

Tabelle 2.

Charakteristik der absoluten Masse (A).

\begin{tabular}{|c|c|c|c|c|c|c|c|}
\hline Nr. & Me $x$ k m a 1 & $\begin{array}{c}\text { Anzah1 } \\
\text { der } \\
\text { Exem- } \\
\text { plare } \\
\text { /N/ }\end{array}$ & $\begin{array}{c}\text { Vertbere1ch } \\
\qquad / 2 /\end{array}$ & $\begin{array}{l}\text { Mitte1- } \\
\text { wert } \\
\text { /x/ }\end{array}$ & $\begin{array}{c}\text { Stan- } \\
\text { dard- } \\
\text { fehler } \\
\text { /S/ }\end{array}$ & $\begin{array}{c}\text { Varia- } \\
\text { t1ons- } \\
\text { koeffi- } \\
\text { z1ent } \\
\text { /v/ }\end{array}$ & $\begin{array}{c}\text { Korelations- } \\
\text { kceefiz1ent } \\
/ \text { mit Werk- } \\
\text { mal } \text { Wr. } 1 / \\
/ r /\end{array}$ \\
\hline 1 & Basion-Ethmoideun & 35 & $52,0-106,0$ & 87,96 & $\pm 13,78$ & 15,66 & \\
\hline 2 & Bas1on-Prostb1 on & 35 & $69,0-226,7$ & 175,26 & $\pm 39,79$ & 22,70 & $+97,7$ \\
\hline 3 & Op1sth1on-Nas1on & 35 & $56,8-135,5$ & $.106,13$ & $\pm 19,55$ & 18,42 & $+97,9$ \\
\hline 4 & Nasion-Prosthion & 35 & $29,5-134,4$ & 100,71 & $\pm 26,55$ & 26,36 & $+95,7$ \\
\hline 5 & Op1sth1on-Sagektotbion & 35 & $50,0-127,0$ & 97,12 & $\pm 18,32$ & 16,87 & $+95,3$ \\
\hline 6 & Sagektorb1 on-Prosth1on & 35 & $44,0-159,0$ & 116,53 & $\pm 28,07$ & 24,09 & $+94,3$ \\
\hline \multirow[t]{2}{*}{7} & Höhe des Trigonum & & & & & & \\
\hline & frontale & 35 & $29,5-99,0$ & 50,71 & $\pm 14,38$ & 28,37 & $-57,6$ \\
\hline 8 & Orbitale-Prosthion & 35 & $23,8-123,5$ & 87,32 & $\pm 23,77$ & 27,22 & $+93,8$ \\
\hline 9 & $2 y g 1$ on $-3 y g 1$ on & 35 & $56,2-136,5$ & 107,53 & $\pm 18,87$ & 17,55 & $+76,8$ \\
\hline \multirow[t]{2}{*}{10} & Grösste Bre1te des & & & & & & \\
\hline & Trigonum occipitale & 35 & $33,3-89,6$ & 67,45 & $\pm 12,43$ & 18,43 & $+92,6$ \\
\hline 11 & Euryon-Euryon & 35 & $44,5-69,0$ & 58,18 & $\pm 5,62$ & 9,65 & $+68,8$ \\
\hline 12 & $\begin{array}{l}\text { Prontostenion-Fronto- } \\
\text { stenion }\end{array}$ & 35 & $39,8-50,0$ & $4,0,28$ & $+5,16$ & 12,82 & $+30,0$ \\
\hline 13 & Ektorb1tale-Ektorbitale & 35 & $38,8-.78,0$ & 58,10 & $\pm 12,31$ & 21,19 & $+72,7$ \\
\hline 14 & Bntorb1tale-Entorb1tale & 35 & $22,6-57,0$ & 40,87 & $\pm 9,25$ & 22,64 & $+78,6$ \\
\hline 15 & Kle1nste Gaumenbre1te & 35 & $16,5-50,3$ & 36,27 & $\pm 8,15$ & 22,48 & $+71,0$ \\
\hline 16 & H1rnschädelhöhe & 35 & $40,4-70,0$ & 56,27 & $\pm 7,24$ & 12,86 & $+71,7$ \\
\hline 17 & Nasalhöhe & 35 & $10,6-34,0$ & 26,69 & $\pm 5,77$ & 21,62 & $+83,4$ \\
\hline 18 & Länge der Backzahnre1he & 34 & $20,2-86,5$ & 66,38 & $\pm 15,04$ & 22,65 & - \\
\hline 19 & Länge der Molarrethe & 34 & $6,3-26,0$ & 20,77 & $\pm 4,20$ & 20,21 & - \\
\hline 20 & Länge der Prämolarre1he & 34 & $14,2-56,5$ & 50,39 & $\pm 11,34$ & 22,50 & - \\
\hline 21 & Länge des Reisszahnes & 33 & $11,0-22,0$ & 18,76 & $\pm 2,27$ & 12,12 & - \\
\hline
\end{tabular}

ihm hoch korrelierten Masse $(2-6,8)$ schliessen lässt. Schliesslich ist $B-P$ sehr hoch $(r=99,1 \%)$ mit $N-P$ korreliert, also mit einem Mass, das im Vergleich zu $O-N$ einen labilen Parameter des Schädels darstellt.

Aus diesen Beobachtungen folgt, dass $B-E$ thm. sehr gute Auskunft über die Grösse des Tieres und $B-P$ über den von der Entwicklung abhängenden Gesichtsteil der Schädellänge gibt. Es sei noch gesagt, dass eine mit der von $N-P$ gleichgrosse Labilität die beiden übrigen Längenmasse des Gesichtsschädels $(6,3)$ und die Höhe des Trigonum frontale $(7)$ 
aufweisen, was im Gegensatz zu dem Verhalten der Längenmasse des Hirnschädels $(1,3,5)$ steht.

1. Innere Hirnhöhlenlänge $(B-E$ thm.): Ein sehr gutes Merkmal, informiert vorzüglich über die Grösse des Hundes, weist eine sehr hohe positive Korrelation mit der Widerristhöhe (Abb. 2), sowie mit allen anderen Längenmassen des Schädels $(2-6,8)$, der grössten Breite des Trigonum occipitale (10) und dem Längen-Breiten-Index (201) auf. Sie kann vorteilhaft zur Einteilung der Schädel in Grössenklassen der Hunde benützt werden, wie folgt: I - sehr kleine Tiere (z.B. Ratle Pinscher), Werte bis $65,0 \mathrm{~mm}$; II - kleine Tiere (z.B. Grossspitz Nr. 4), Werte 65,1-75,0 mm; III - mittelgrosse Tiere (z.B. Polnischer Tiefland-Schäferhund Nr. 8), Werte $75,1-85,0 \mathrm{~mm}$; IV - grosse Tiere (z.B. Airedale Terrier), Werte $85,1-95,0 \mathrm{~mm}$; V - sehr grosse Tiere (z.B. Bernhardiner Nr. 28) mit Werten über $95 \mathrm{~mm}$.

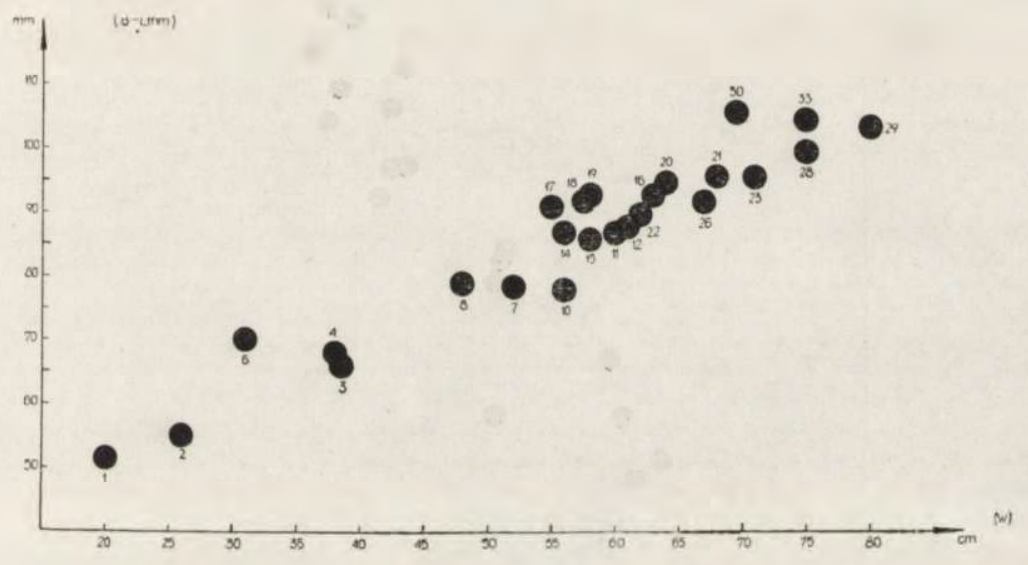

Abb. 2. Abhängigkeit der inneren Hirnhöhlenlänge (B-Ethm.) von der Widerristhöhe $(W)$ des Hundes.

2. Basailänge $(B-P)$ : Ein gutes Merkmal, sehr hoch mit den Längenmassen $(1,3-6,8)$, der grössten Breite des Trigonum occipitale (10) und dem Längen-Breiten-Index (201) korreliert (Tabelle 3, Abb. 3). Einen grossen Einfluss auf die Werte dieses Merkmals hat im Bereich der Tiere ähnlicher Grösse das Mass $N-P$, mit dem es hoch korreliert ist und dessen grosse Variabilität $(V=26,4 \%)$ die grosse Variabilität des besprochenen Merkmals verursacht. Mit diesem Merkmal kann eine Einteilung der Hundeschädel in die folgenden Klassen erfolgen: I - sehr kurze mit Werten bis $95,0 \mathrm{~mm}$ (z.B. Ratle Pinscher), II - kurze: 95,1-135,0 mm (z.B. Großspitz Nr. 4), III - mittellange: $135,1-175,0 \mathrm{~mm}$ (z.B. Polnischer Tiefland-Schäferhund Nr. 8), IV - lange: $175,1-215,0 \mathrm{~mm}$ (z.B. 
Bergland-Schäferhund), V — sehr lange: Werte über $215 \mathrm{~mm}$ (z.B. Barsoi Nr. 35).

b) Die Breitenmasse (9-15) sind im Vergleich zu den Längenmassen weniger stark untereinander korreliert (Tabelle 3). Sie können in dieser Beziehung in 3 Gruppen geteilt werden: zur ersten gehören Masse (wie z. B. die grösste Breite des Trigonum occipitale), die mit gewissen Längenmassen $(B-P, B-E t h m ., O-N, O-$ Sagekt) hoch korreliert sind,

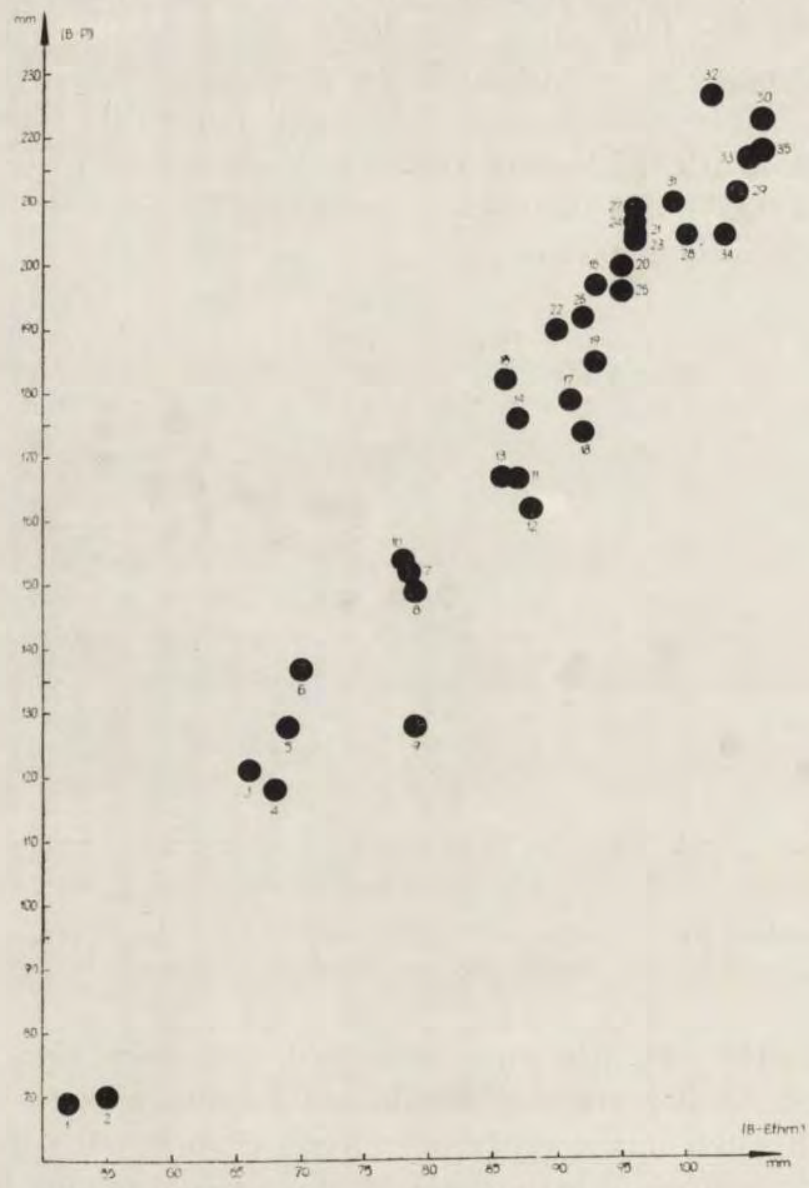

Abb. 3. Abhängigkeit der Basallänge $(B-P)$ von der inneren Hirnhöhlenlänge (B-Ethm.).

zur zweiten Gruppe gehören Masse, die von anderen Parametern relativ unabhängig sind (wie z. B. $F t-F t$ oder auch $E u-E u$ ), zur dritten Gruppe gehören Masse, die sehr hoch untereinander und mit der Hirnschädelhöhe korreliert sind (z. B. Zy-Zy, Ekt-Ekt, Ent-Ent, kleinste Gaumenbreite). 
Die Variationskoeffizienten der Masse dieser Gruppe betragen von 9,65 bis $22,64 \%$. Die grössten Werte haben die Breitenmasse der Stirngegend $(E n t-E n t, E k t-E k t)$ und des Gesichtsteils, die kleinsten Werte weisen $E u-E u$ und $F t-F t$ auf.

c) Die Höhenmasse. Von den 2 in Betracht gezogenen Markmalen weist die Hirnschädelhöhe eine hohe Korrelation mit allen 4 Breitenmassen (9, 13-15) auf. Die Nasalhöhe verhält sich ähnlich wie die Höhe des Trigonum frontale und $F t-F t$ und weist keine hohen Korrelationen auf (Tabelle 3). Der Variationskoeffizient der Hirnschädelhöhe ist relativ niedrig $(12,9 \%)$, der der Nasalhöhe - relativ hoch $(21,6 \%)$.

d) Die Zahnmasse (18-21). Von diesen haben den relativ höchsten und niedrigsten Variationskoeffizienten entsprechend die Länge der Backzahnreihe $(V=22,7 \%)$ und die Länge des Reisszahnes $(V=12,1 \%)$. Von den zwei übriggebliebenen Zahnmassen ist die Molarreihenlänge stabiler als die Prämolarreihenlänge.

Korrelationen zwischen diesen Merkmalen wurde wegen technischen Schwiergkeiten (das Fehlen von Zähnen in 2 Exemplaren) nicht berechnet.

\section{B. Die absoluten Masse in Prozenten der Basallänge ausgedruickt}

Alle Breiten- und Höhenmasse des Schädels, in Prozenten der Basallänge ausgedrückt, und alle Längenmasse des Hirnschädels dieser Gruppe sind negativ mit den in der gleichen Weise ausgedrückten Längenmassen des Gesichtsschädels korreliert (Tabelle 4).

Der Variationskoeffizient der Parameter dieser Gruppe (Tabelle 5) ist klein für die mit der Basellänge $(B-P)$ korrelierten Längenmasse und gross für die mit der Basallänge nicht korrelierten Breiten- und Höhenmasse. Daraus folgt, dass der taxonomische Wert der Parameter dieser Gruppe durch die Grösse der Korrelation zwischen dem konstanten Nenner $(B-P)$ und dem variablen Zähler gegeben ist. Ist die Korrelation zwischen den Elementen des Parameters hoch, so ist der Variationskoeffizient dieses Parameters klein, ist die Korrelation klein, so ist der Variationskoeffizient gross. Eine der Konsequenzen derartiger Abhängigkeiten ist der kleine taxonomische Wert der Parameter vom ersten Typ (kleines $V$ ) und eine einseitige, aber nicht zulässige Beurteilung des Materials durch die Parameter vom zweiten Typ.

$\mathrm{Zu}$ den stabilen Massen dieser Gruppe gehören die Längenmasse des Schädels (ausser der Höhe des Trigonum frontale), die grösste Breite des Trigonum occipitale und die Nasalhöhe, zu den labilen - die Breitenmasse des Schädels (ausser der grössten Breite des Trigonum occipitale), die Hirnschädelhöhe und die Höhe des Trigonum frontale. 
Eine genauere Charakteristik von drei dieser Parameter sei folgend gegeben (Tabellen $3-5$ ):

1. Jochbogenbreite (109): Dieser Parameter drückt die Relation von $Z y--Z y$ zu $B-P$ aus. Er entstand aus mittelmässig korrelierten $(r=$ $72,7 \%)$ und ziemlich variablen $\left(V_{9}=17,6 \%, V_{2}=22,7 \%\right)$ Merkmalen. Der Index informiert über die Variabilität des Schädels in der Stirnebene. Er ist hoch positiv mit den folgenden Parametern der Gruppe B (Tabelle 4) korreliert: Grösste Breite des Trigonum occipitale (110), kleinste

Tabelle 5 .

Charakteristik der absoluten Masse die in Prozenten der Basallänge ausgedrückt sind (B).

\begin{tabular}{|c|c|c|c|c|c|c|c|}
\hline Nr. & Me $\mathrm{rk}$ m a $\mathrm{l}$ & $\begin{array}{c}\text { Anzahl } \\
\text { der } \\
\text { Exem- } \\
\text { plare } \\
\text { /N/ }\end{array}$ & $\begin{array}{l}\text { Wertbereich } \\
\qquad / 2 /\end{array}$ & $\begin{array}{l}\text { M1ttel- } \\
\text { wert } \\
\mid \bar{x} /\end{array}$ & $\begin{array}{l}\text { Stan- } \\
\text { dard- } \\
\text { fehler } \\
\text { /S/ }\end{array}$ & $\begin{array}{l}\text { Varia- } \\
\text { t1ons- } \\
\text { koeff1- } \\
\text { z1ent } \\
/ \mathrm{v} /\end{array}$ & $\begin{array}{c}\text { Korelations- } \\
\text { koeffiz1ent } \\
\text { /mit Merk- } \\
\text { mal Ifr. } 2 / \\
/ \mathrm{r} /\end{array}$ \\
\hline 101 & Bas1on-Ethmo1 deum & 35 & $44,99-78,57$ & 51,61 & $\pm 7,13$ & 13,82 & $+97,7$ \\
\hline 103 & Op1sth1 on-Nasion & 35 & $56,94-84,06$ & 61,65 & $\pm 6,12$ & 9,92 & $+97,8$ \\
\hline 104 & Nas1on-Prosthion & 35 & $41,71-61,96$ & 56,72 & $\pm 4,19$ & 7,39 & $+99,1$ \\
\hline 105 & Op1sth1on-Sagektorb1on & 35 & $47,52-76,38$ & 56,43 & $\pm 5,97$ & 10,59 & $+94,6$ \\
\hline 106 & Sagektorb1 on-Prosth1 on & 35 & $59,37-72,94$ & 66,34 & $\pm 3,47$ & 5,23 & $+97,7$ \\
\hline \multirow[t]{2}{*}{107} & Höhe des Trigonum & & & & & & \\
\hline & frontale & 35 & $14,22-143,5$ & 33,85 & $\pm 26,43$ & 78,07 & $-61,5$ \\
\hline 108 & Orb1 tale-Prosth1on & 35 & $34,00-57,35$ & 49,10 & $\pm 4,42$ & 9,01 & $+97,7$ \\
\hline 109 & zyg1on-zyg1on & 35 & $46,56-101,4$ & 63,43 & $\pm 12,29$ & 19,37 & $+72,7$ \\
\hline \multirow[t]{2}{*}{110} & Grösste Bre1te des & & & & & & \\
\hline & Tr1gonum oco1pitale & 35 & $33,21-58,00$ & 39,30 & $\pm 4,88$ & 12,13 & $+92,1$ \\
\hline 111 & Buryon-Buryon & 35 & $25,83-65,43$ & 35,14 & $\pm 9,21$ & 26,23 & $+66,0$ \\
\hline \multirow[t]{2}{*}{112} & Frontostenion-Fronto- & & & & & & \\
\hline & sten1on & 35 & $15,60-62,75$ & 24,80 & $\pm 9,63$ & 38,83 & $+22,8$ \\
\hline 113 & Bktorb1tale-Bktorb1tale & 35 & $24,17-57,34$ & 34,30 & $\pm 8,19$ & 23,88 & $+66,4$ \\
\hline 114 & Entorb1tale-Entorb1tale & 35 & $17,66-38,83$ & 23,87 & $\pm 4,74$ & 19,87 & $+74,4$ \\
\hline 115 & Kle1nste Gaumenbre1te & 35 & $14,68-36,72$ & 21,21 & $\pm 4,44$ & 20,93 & $+67,4$ \\
\hline 116 & H1 rnschädelhöhe & 35 & $24,64-38,57$ & 33,71 & $\pm 7,99$ & 23,72 & $+68,4$ \\
\hline 117 & Nasalhöhe & 35 & $12,62-23,57$ & 15,45 & $\pm 2,28$ & 14,75 & $+83,8$ \\
\hline 118 & Länge der Backzahnreihe & 34 & $29,28-42,95$ & 37,41 & $\pm 2,58$ & 6,91 & - \\
\hline 119 & Länge der Nolarre1he & 34 & $9,13-14,71$ & 11,83 & $\pm 1,26$ & 10,69 & - \\
\hline 120 & Länge der Pränolarre1he & 34 & $20,58-31,81$ & 28,43 & $\pm 2,14$ & 7,54 & - \\
\hline 121 & Länge de 3 Re1sszahnes & 33 & $8,78-15,71$ & 10,65 & $\pm 1,41$ & 13,30 & - \\
\hline
\end{tabular}

Gaumenbreite (115), Hirnschädelhöhe (116) und Stirnbreite (113). Die Schädel können mittels dieses Indexes, wie Abb. 4 schematisch zeigt, in folgende Klassen geteilt werden: I - sehr schmal (z. B. Barsoi Nr. 35), II - schmall (z. B. Bernhardiner Nr. 27), III - mittelbreit (z. B. Grossspitz Nr. 4), IV - breit (z. B. Boxer Nr. 12), V - sehr breit (z. B. Bulldogge).

2. Hirnschädelbreite (111): Dieser Parameter entstand als Relation von $E u-E u$ zu $B-P$, also aus relativ schwach korrelierten $(r=66,0 \%)$ und 
unterschiedlichen hinsichtlich ihrer Variabilität $\left(V_{11}=9,7 \%, V_{2}=22,7 \%\right)$ Parametern. Hohe Korrelation existiert mit den folgenden Parametern:

(a) der Gruppe B (positiv korreliert): Hirnschädelhöhe (116), innere Hirnhöhlenlänge (101), Schädellänge (112), Höhe des Trigonum frontale (107), (b) der Gruppen A und C (negativ korreliert): Or $-P$, Sagekt $-P$, $N-P, B-P$, Längen-Breiten-Index des Hirnschädels (201). Wie aus

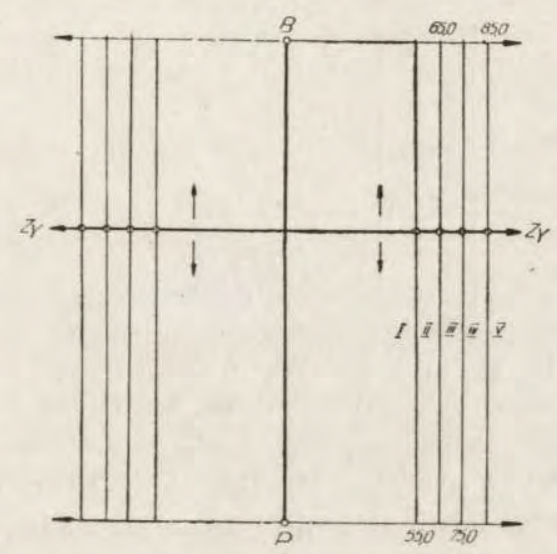

Abb. 4. Schematische Darstellung der Werte der Jochbogenbreite in Prozenten der Basallänge ausgedrückt (109) and der Einteilung in Klassen I-V.

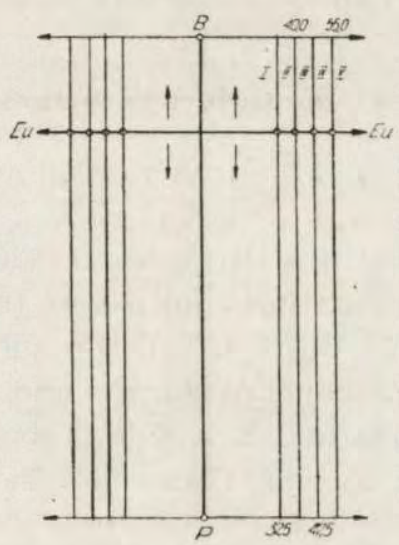

Abb. 5. Schematische Darstellung der Werte der Schädelbreite in Prozenten der Basallänge ausgedrückt (111) und die Einteilung in Klassen I-V.

Abb. 5 ersichtlich, können mit Hilfe dieses Parameters die Schädel hinsichtlich der Gestalt des Hirnschädels auf folgende Weise gegliedert werden: I - sehr schmal (z. B. Barsoi Nr. 35), II - schmal (z. B. Deutsch Drahthaar), III - mittelbreit (z. B. Grossspitz Nr. 4), IV - breit (z. B. 3ulldogge), V - sehr breit (z. B. Pekinese). 
3. Hirnschädelhöhe (116): Dieser Parameter ist als Relation der Hirnschädelhöhe zu $B-P$ ausgedrückt. Er entstand aus relativ niedrig korrelierten $(r=68,4 \%)$ und hinsichtlich der Variabilität verschiedenen $\left(V_{16}=12,9 \%, V_{2}=22,7 \%\right)$ Parametern und ist hoch positiv mit den folgenden Parametern der Gruppe B korreliert Schädelbreite: (111), -enge (112), anatomische (105) und morphologische (103) Cranialachse, innere

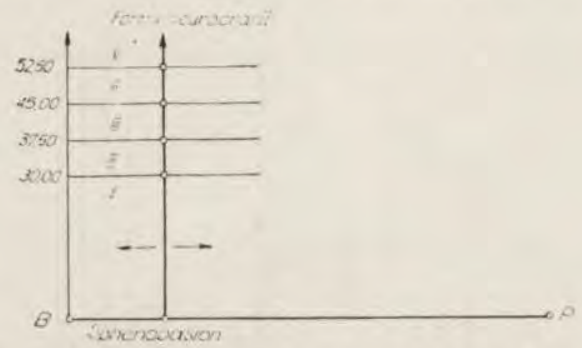

Abb. 6. Schematische Darstellung der Werte der Hirnschädelhöhe in Prozenten der Basallänge ausgedrückt (116) und die Einteilung in Klassen I-V.

Hirnhöhlenlänge (101), grösste Breite des Trigonum occipitale (110), Jochbogenbreite (109). Abb. 6 zeigt die vorgeschlagene Einteilung der Schädel mittels dieses Parameters: I - sehr niedrig (z. B. Barsoi Nr. 35), II -- niedrig (z. B. Polnischer Tiefland-Schäferhund Nr. 7), III - mittelhoch (z. B. Grossspitz Nr. 4), IV - hoch (z. B. Bulldogge), V - sehr hoch (z. B. Pekinese).

\section{Die Quotienten-Indizes}

Es wurden die folgenden 11 Parameter dieser Gruppe betrachtet Tabellen $3,4,6)$ :

1. Längen-Breiten-Index des Hirnschädels (201): Die Bestandteile dieses Parameters sind ziemlich hoch korreliert $(r=80,5 \%)$ und verschieden variabel $\left(V_{5}=18,9 \%, V_{11}=9,7 \%\right)$. Er gibt über die Gestalt des Hirnschädels in der Stirnebene Auskunft und informiert auch vorzüglich uber alle Längenmasse des Schädels (Tabelle 3), dadurch aber wieder über $B-E$ thm auch über die Grösse des Tieres (Abb. 7). Die grössten Werte nimmt dieser Index bei grossen Hunden mit sehr langen Hirnschädel an, was bedeutet, dass sich die Gestalt der Hirnhöhle dieser Tiere durch Verlängerung bei gleichbleibender Breite und Höhe ändert. Abb. 8 zeigt, wie die Hundeschädel mit Hilfe dieses Parameters in I - sehr wenig, II - wenig, III - mittelmässig, IV - hoch und V - sehr hoch längenmässig ausgestreckte, entsprechend bei sehr kleinen (z. B. Ratle Pinscher), kleinen (z. B. Grossspitz Nr. 4), mittleren (z. B. Polnischer Tiefland-Schäferhund Nr. 3), grossen (z. B. Airedale Terrier) und sehr grossen (z. B. Polnischer Bergland-Schäferhund) Tieren (Abb. 9). 
Tabelle 6.

Charakteristik der Quotienten-Indizes (C).

\begin{tabular}{|c|c|c|c|c|c|c|c|c|}
\hline Nr. & In $d \theta x$ & $\begin{array}{c}\text { Anzahl } \\
\text { der } \\
\text { Exem- } \\
\text { plare } \\
\text { /N/ }\end{array}$ & Wertb & $\begin{array}{l}\text { bere1ch } \\
\mid z /\end{array}$ & $\begin{array}{l}\text { Mittel- } \\
\text { wert } \\
\mid \bar{x} /\end{array}$ & $\begin{array}{r}\text { Stan- } \\
\text { dard- } \\
\text { Pehler } \\
\text { /S/ }\end{array}$ & $\begin{array}{l}\text { Var1a- } \\
\text { t1ons- } \\
\text { kooff1- } \\
\text { z1ent } \\
/ \mathrm{v} /\end{array}$ & $\begin{array}{l}\text { Korelations- } \\
\text { koeffizient } \\
\text { zw1schen } \\
\text { zahler und } \\
\text { Nenner /r/ }\end{array}$ \\
\hline 201 & $\begin{array}{l}\text { Längen-Bre1ten-Index } \\
\text { des H1rnsonädels }\end{array}$ & 35 & 109,17 & $-199,66$ & 165,89 & $\pm 22,53$ & 13,58 & $+80,5$ \\
\hline 202 & St1rndre1eck-Index & 35 & 52,21 & $-255,15$ & $\therefore, 19$ & $\pm 44,35$ & 47,59 & $-14,4$ \\
\hline 203 & St1rnbre1 ten-Index & 35 & 24,17 & $-57,34$ & 34,40 & $\pm 8,19$ & 23,88 & $+66,4$ \\
\hline 204 & Höhendifferenz-Index & 35 & 163,91 & $-381,13$ & 218,25 & $\pm 40,11$ & 18,38 & $+79,6$ \\
\hline 205 & St1rnlage-Index & 35 & 83,79 & $-153,47$ & 118,99 & $\pm 14,99$ & 12,60 & $+88,5$ \\
\hline $\begin{array}{l}206 \\
207\end{array}$ & $\begin{array}{l}\text { Bre1tend1fferenz-Index } \\
\text { Höhen-Iängen-Index }\end{array}$ & 35 & 267,92 & $-340,61$ & 300,60 & $\pm 21,10$ & 7,02 & $+97,4$ \\
\hline 208 & $\begin{array}{l}\text { des H1rnschädels } \\
\text { Ausprägungs-Index }\end{array}$ & 35 & 51,89 & $-83,07$ & 64,74 & $\pm 7,42$ & 11,46 & $+71,7$ \\
\hline 209 & $\begin{array}{l}\text { des H1rnsohädels } \\
\text { Bre1ten-Höhen-Index }\end{array}$ & 35 & 102,77 & $-195,20$ & 145,83 & $\pm 15,63$ & 10,72 & $+63,9$ \\
\hline & des H1rnschädels & 35 & 88,06 & $-120,43$ & 104,01 & $\pm 6,29$ & 6,29 & $+88,1$ \\
\hline 210 & Backzahn-Index & 34 & 101,49 & $-127,74$ & 107,74 & $\pm 4,55$ & 4,23 & - \\
\hline 211 & Re1sszahn-Index & 33 & 78,08 & $-125,00$ & 89,51 & $\pm 8,44$ & 9,43 & - \\
\hline
\end{tabular}

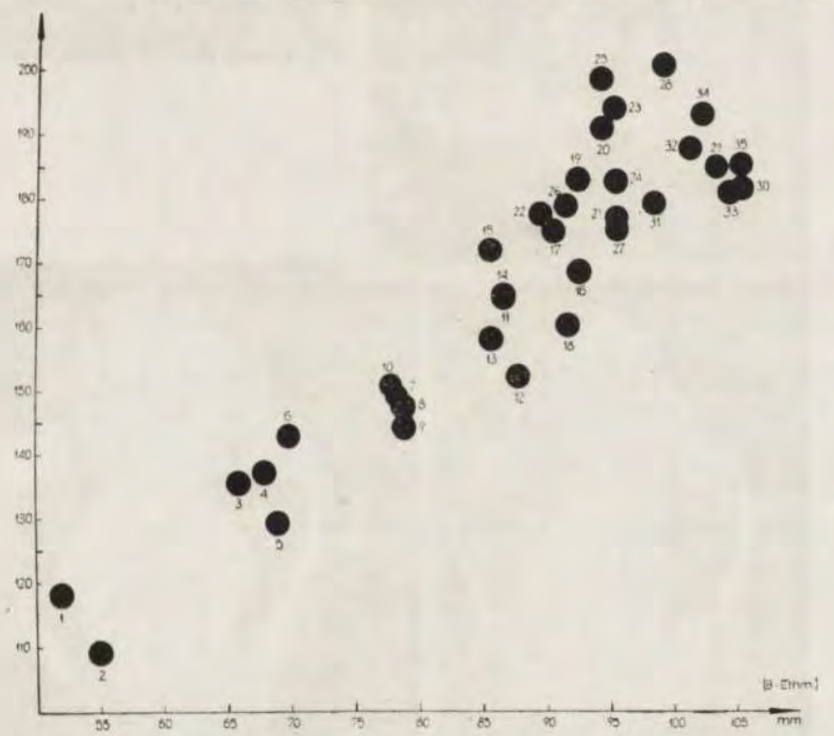

Abb. 7. Abhängigkeit des Längen-Breiten-Indexes des Hirnschädels (201) von der inneren Hirnhöhlenlänge $(B-E$ thm.).

2. Stirndreieck-Index (202): Dieser Parameter entstand als einziger aus Massen, die negativ sehr wenig korreliert $(r=-14,4 \%)$, aber sehr hoch variabel $\left(V_{7}=28,4 \%, V_{13}=21,2 \%\right)$ sind. Er informiert über den Charakter des Trigonum frontale, dessen Höhe negativ von der Entwicklung der $M m$. temporales abhängt. Er nimmt die kleinsten Werte bei klei- 


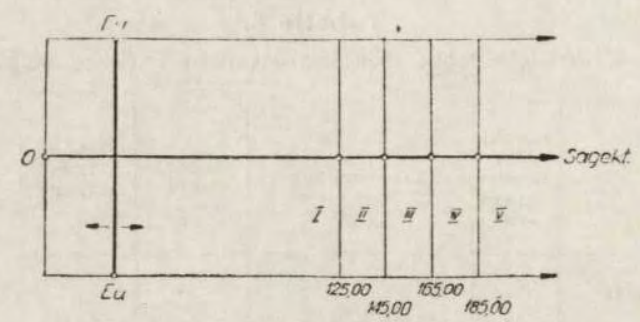

Abb. 8. Schematische Darstellung der Werte des Längen-Breiten-Indexes des Hirnschädels (201) und die Einteilung in Klassen I-V.
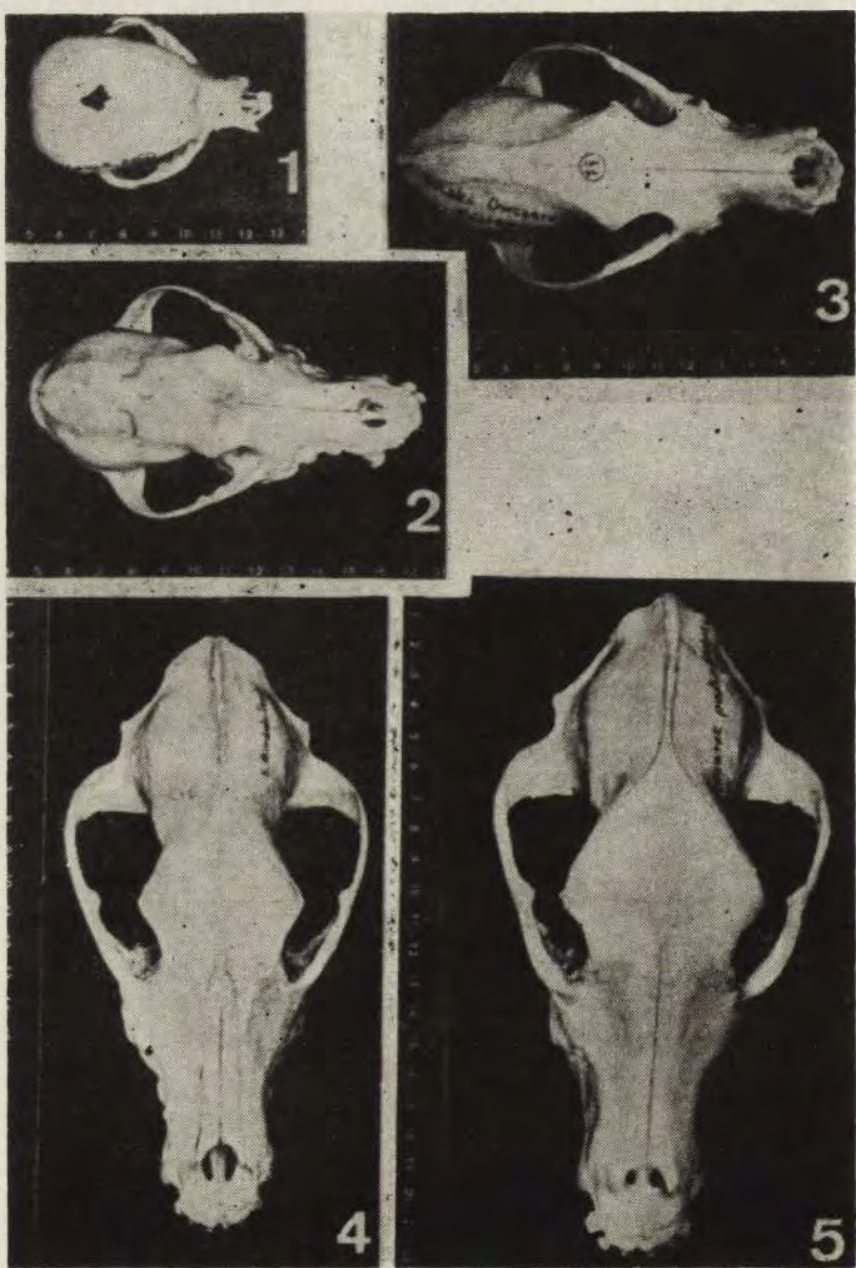

Abb. 9. Eine Illustration der Werte des Längen-Breiten-Indexes des Hirnschädels bei: 1-Ratle Pinscher Nr. 1, 2-Grossspitz Nr. 4, 3-Polnischen Tiefland-Schäferhund, 4 - Airedale-Terrier, 5 - Polnischen Bergland-Schäferhund. 
nen Hunden (negative Korrelation mit B-Ethm) an. Er teilt die Schädel in solche mit: I - sehr niedrigen (z. B. Deutscher Schäferhund Nr. 21), II - niedrigen (z. B. Polnischer Bergland-Schäferhund), III - mittelhohen (z. B. Polnischer Tiefland-Schäferhund), IV - hohen (ein Beispiel in unserem Material fehlt), und V - sehr hohen (z. B. Grossspitz Nr. 4) Stirndreiecken (Abb. 10). Für Schädel, die kein Stirndreieck aufweisen, nimmt dieser Index sehr grosse Werte an, so z. B. beim Ratle-Pinscher den Wert 255,15.

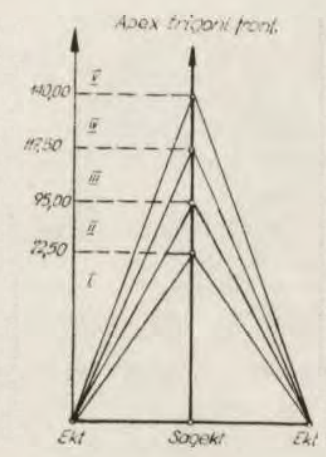

Abb. 10. Schematische Darstellung der Werte des Stirndreieck-Indexes (202) und die Einteilung in Klassen I-V.

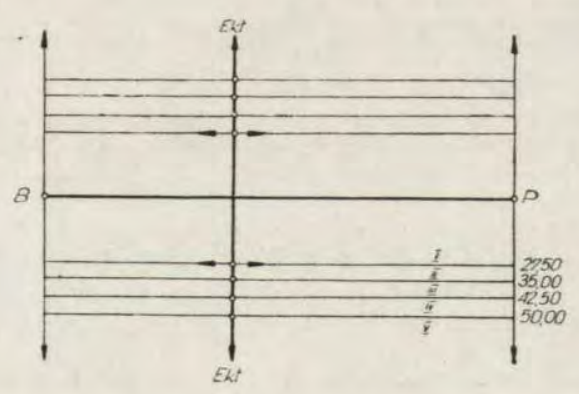

Abb. 11. Schematische Darstellung der Werte des Stirnbreiten-Indexes (203) und die Einteilung in Klassen $\mathrm{I}-\mathrm{V}$.

3. Stirnbreiten-Index (203): Entstand aus Massen relativ unhoch korrelierten $(r=66,4 \%)$ und ziemlich variablen $\left(V_{13}=21,2 \%, V_{2}=22,7 \%\right)$. Informiert über die Gestalt des Schädelhauptteils (ohne den Jochbögen) in der Stirnebene. Er nimmt die grössten Werte bei kurzschnauzigen (negative Korrelation mit dem Stirnlage-Index) Hunden an. Wie Abb. 11 zeigt, können mit Hilfe dieses Indexes die Schädel wie folgt eingeteilt werden: I - sehr engstirnig (z. B. Barsoi Nr. 35), II - engstirnig (z. B. 
Airedale Terrier), III - mittelstirnig (z. B. Bernhardiner Nr. 28), IV breitstirnig (z. B. Boxer Nr. 12), V - sehr breitstirnig (z. B. Bulldogge).

4. Höhendifferenz-Index (204): Dieser Parameter entstand aus Massen, die mittelmässig abhängig $(r=79,6 \%)$ und sehr verschieden hinsichtlich ihrer Variabilität gestaltet sind $\left(V_{15}=12,9 \%, V_{17}=21,6 \%\right)$. Nimmt die grössten Werte bei kurzköpfigen Hunden an (negative Korrelation mit $B-P$ ). Er informiert über das Schädelseitenprofil und

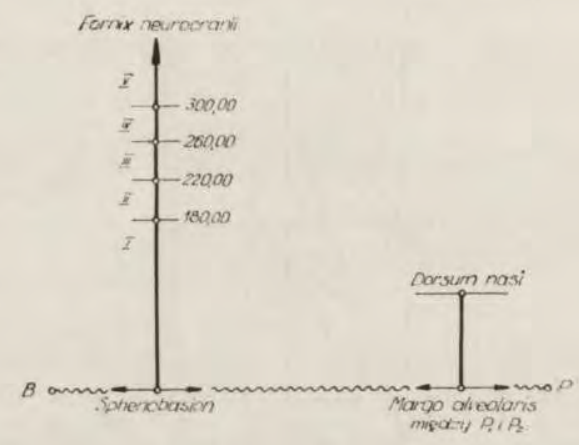

Abb. 12. Schematische Darstellung der Werte des Höhendifferenz-Indexes (204) und die Einteilung in Klassen I-V.

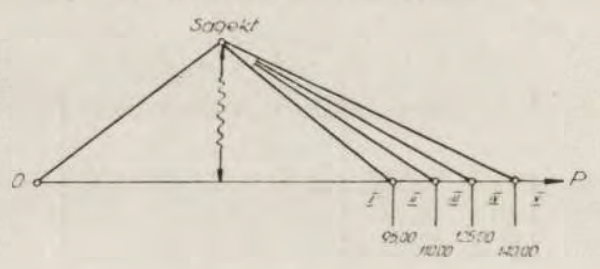

Abb. 13. Schematische Darstellung der Werte des Stirnlage-Indexes (205) und dic Einteilung in Klassen I-IV.

kann zur Einteilung der Schädel in folgende Klassen benützt werden (Abb. 12): I - mit sehr kleinen (z. B. Deutscher Schäferhund Nr. 21), II - mit kleinen (z. B. Barsoi Nr. 35), III - mit mittelmässigen (z. B. Folnischer Tiefland-Schäferhund $\mathrm{Nr} 8$ ), IV - mit grossen (z. B. Grossspitz Nr. 4) und V - mit sehr grossen Höhendifferenzen (z. B. Ratle Finscher). Dieser Index kann in einem gewissen Grad die Wagnersche Gesichtskrümmung (W a g n e r, 1929) ersetzen.

5. Stirnlage-Index (205) (B rin kma n n, 1921): Entstand aus Massen ziemlich hoch korrelierten $(r=88,5 \%)$ und an Variabilität sehr verschiedenen $\left(V_{6}=24,1 \%, V_{5}=18,9 \%\right)$. Gibt gute Auskunft über die Länge der Schnauze, nimmt seine grössten Werte bei engstirnigen Hunden an (negative Korrelation mit dem Stirnbreiten-Index). Wie Abb. 13 
zeigt, kann dieser Parameter zur Einteilung der Schädel in folgende Klassen benützt werden: I - sehr kurzschnauzig (z. B. Bulldogge), II kurzschnauzig (z. B. Boxer Nr. 12), III - mittellangschnauzig (z. B. Polnischer Tiefland-Schäferhund Nr. 8), IV - langschnauzig (z. B. Deutscher
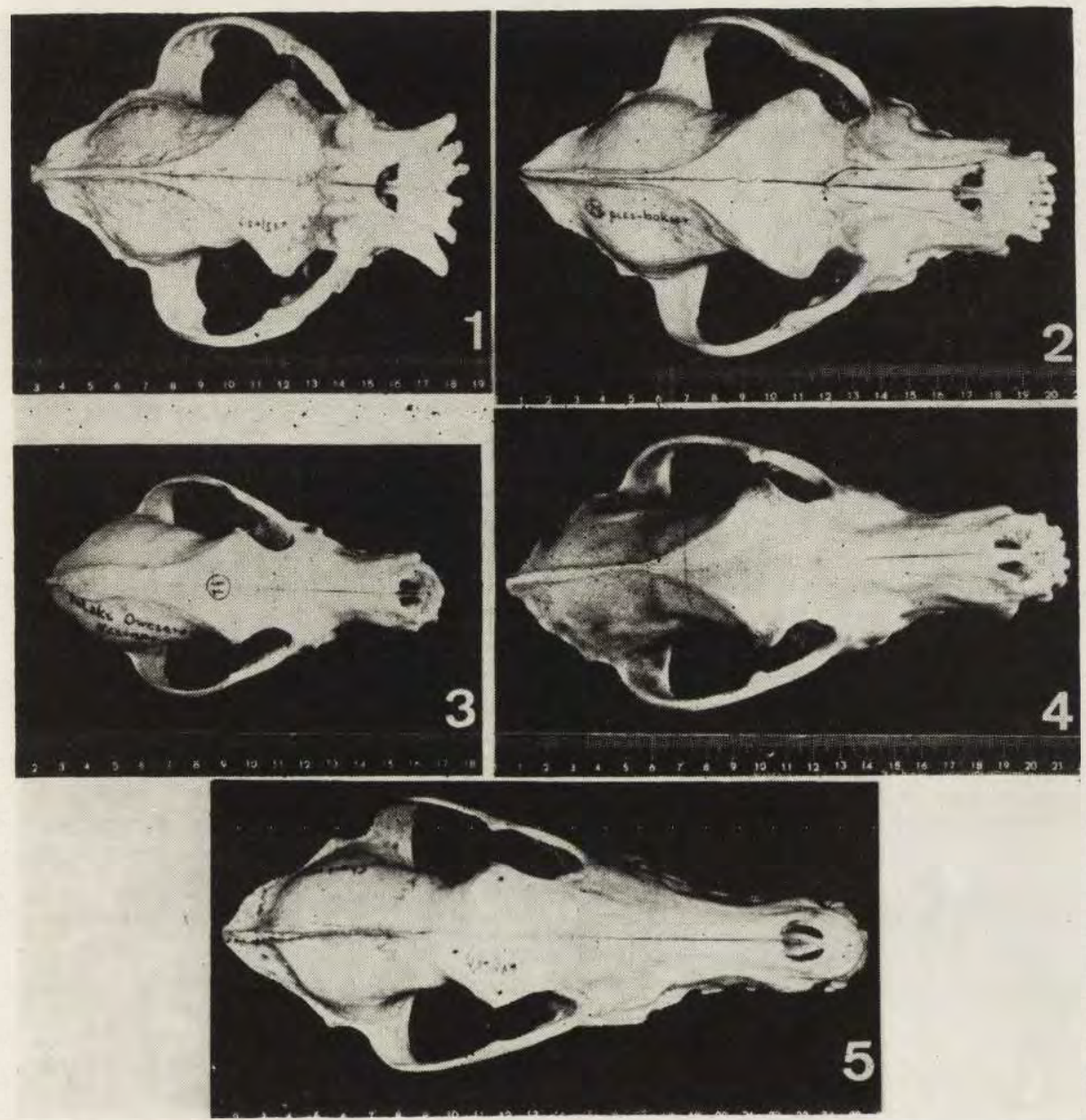

Abb. 14. Eine Illustration der Werte des Stirnlage-Indexes (205): 1 - sehr kurzschnauzig (Bulldogge Nr. 9), 2 - kurzschnauzig (Boxer Nr. 12), 3 - mittellangschnauzig (Polnischer Tiefland-Schäferhund Nr. 8), 4-langschnauzig (Deutscher Schäferhund Nr. 20), 5 - sehr langschnauzig (Barsoi Nr. 35).

Schäferhund Nr. 20) und V - sehr langschnauzig (z. B. Barsoi Nr. 35) (Abb. 14).

6. Breitendifferenz-Index (206): Dieser Index besteht aus Parametern, die hinsichtlich der Variabilität verschiedenartig gestaltet $\left(V_{9}=17,6 \%\right.$, 
$\left.V_{15}=22,5 \%\right)$, aber gleichzeitig sehr hoch korreliert $(r=97,4 \%)$ sind. Deswegen hat er eine sehr kleine Variabilität $\left(V_{206}=7,0 \%\right)$ und sein Wert in der morphologischen Schädeldiagnostik der Gattung Hund ist begrenzt. Er informiert über die Gestalt des Schädels in der Stirnebene und teilt die Schädel in folgende Klassen (Abb. 15): I - mit sehr kleinen, II - mit kleinen, III - mit mittelmässigen, IV - mit grossen, V mit sehr grossen Breitendifferenzen.

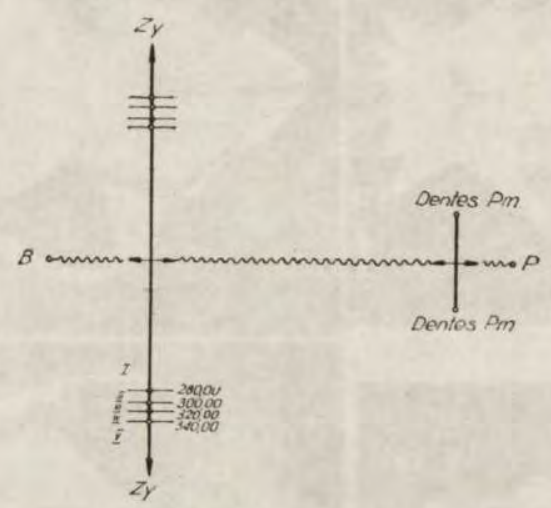

Abb. 15. Schematische Darstellung der Werte des Breitendifferenz-Indexes (206) und die Einteilung in Klassen I-V.

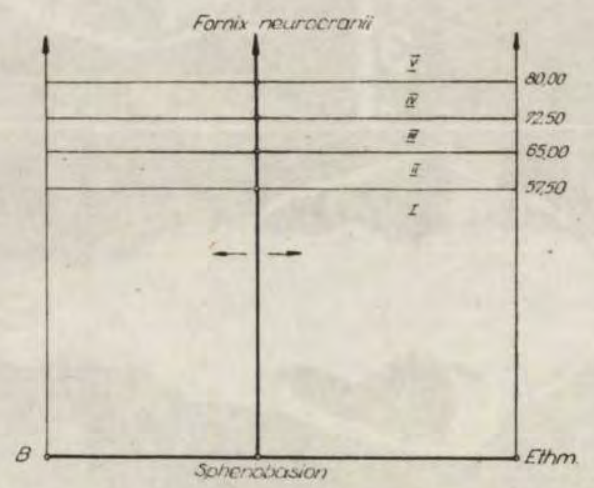

Abb. 16. Schematische Darstellung der Werte des Höhen-Längen-Indexes des Hirnschädels (207) und die Einteilung in Klassen I-V.

7. Höhen-Längen-Index des Hirnschädels (207): Entstand aus wenig variablen $\left(V_{16}=12,9 \%, V_{1}=15,7 \%\right)$ und relativ wenig abhängigen $(r=71,7 \%)$ Massen. Gibt über die Gestalt des Hirnschädels in der Saggittalebene Auskunft. Nimmt die grössten Werte bei kurzschnauzigen Hunden an (negative Korrelation mit dem Stirnlage-Index). Differenziert die 
Schädel in solche mit (Abb. 16): I - sehr niedrigen (z. B. Collie Nr. 23), II - niedrigen (z. B. Irish Setter Nr. 15), III - mittelhohen (z. B. Grossspitz Nr. 4), IV - hohen (z. B. Ratle Pinscher) und V - sehr hohen Hirnschädel (z. B. Boxer Nr. 10).

8. Ausprägungs-Index des Hirnschädels (208): Er entstand aus relativ wenig korrelierten $(r=63,9 \%)$ und ziemlich stabilen $\left(V_{11}=9,7 \%, V_{12}=\right.$ $=12,8 \%)$ Massen. Informiert über den Ausprägungsgrad des Hirnschädels auf die beiden Seiten, also in der Stirnebene. Erreicht seinen maximalen Wert bei engstirnigen Hunden (negative Korrelation mit dem Stirnbreiten-Index). Er kann zur Einteilung der Schädel in solche mit (Abb. 17): I - sehr wenig (z. B. Pekinese), II - wenig (z. B. Bernhardiner Nr. 26), III - mittelmässig (z. B. Irish Setter Nr. 16), IV - gut (z. B. Collie Nr. 24) - und V - sehr gut (z. B. Polnischer Tiefland-Schäferhund Nr. 7) auf die Seiten ausgeprägten Hirnschädel.

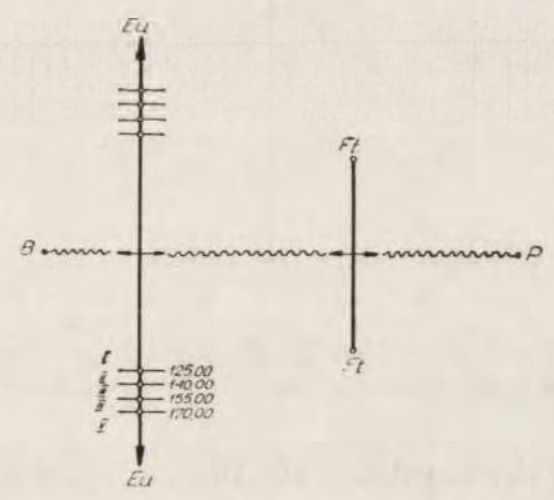

Abb. 17. Schematische Darstellung der Werte des Ausprägungs-Indexes (208) und die Einteilung in Klassen I-V.

9. Breiten-Höhen-Index des Hirnschädels (209): Dieser Parameter entstand aus sehr schwach variablen $\left(V_{11}=9,7 \%, V_{16}=12,9 \%\right)$ und ziemlich hoch korrelierten $(r=88,1 \%)$ Merkmalen. Deshalb hat er selbst eine sehr kleine Variabilität $\left(V_{209}=6,3 \%\right)$ und einen begrenzten Wert für die morphologische Diagnostik des Hundeschädels. Gibt über die Gestalt des Hirnschädels in der Querebene Auskunft und teilt die Schädel in folgende Typen (Abb. 18) ein: I - mit sehr engen, II - mit engen, III mit mittelbreiten, IV - mit breiten und V - mit sehr breiten Hirnschädel.

10. Backzahn-Index (210): Entstand aus ziemlich unstabilen Parametern deren Variationskoeffizienten von $20,2 \%$ bis $22,7 \%$ betragen. Der Index soll über die Art der Linienführung des Backenteils des Zahnbogens informieren. Wegen seiner sehr schwachen Variabilität $\left(V_{210}=4,2 \%\right)$ 
ist jedoch sein Wert für die morphologische Diagnostik des Hundeschädels gering. Seine Korrelation mit anderen Parametern wurde nicht berechnet da in 2 Schädeln die Zähne fehlten.

11. Reisszahn-Index (211): Seine Bestandteile sind sehr verschieden hinsichtlich ihrer Variabilität gestaltet $\left(V_{21}=12,1 \%, V_{19}=20,2 \%\right)$. Informiert über die Entwicklung des Reisszahns. Wie aus dem, Schrifttum (Bogolubskij, 1928; Wyrost \& Kucharczyk, 1967b) und aus unseren, in dieser Arbeit gemachten, Beobachtungen hervorgeht, ist dieser Index bei zeitgenössischen Hunden in der Regel kleiner als 100

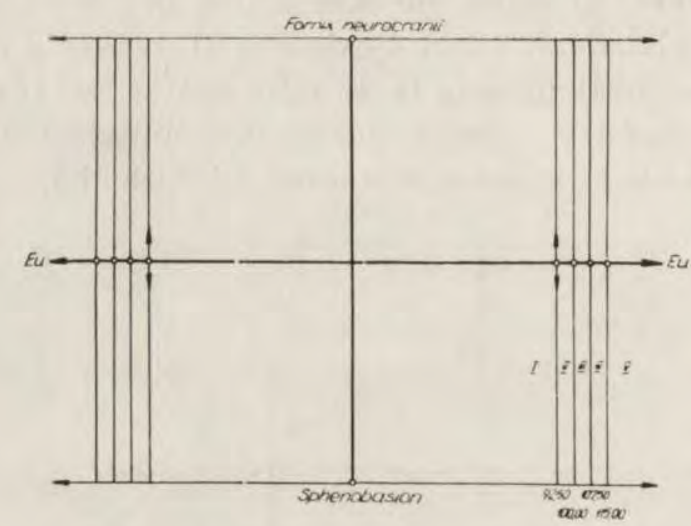

Abb. 18. Schematische Darstellung der Werte des Breiten-Höhen-Indexes des Hirnschädels (209) und die Einteilung in Klassen I-V.

$(\bar{x}=89,5)$ und bei Wölfen grösser als $100(\bar{x}=101,4)$ oder zumindestens ganz nahe von 100. Er kann demnach in gewissen Masse als Hilfsmittel für die Unterscheidung von wolfsähnlichen Hunde- und reinen Wolfsschädeln benützt werden.

\section{BESPRECHUNG DER UNTERSUCHUNGSERGEBNISSE}

\section{A. Die absoluten Masse}

Wie aus Tabelle 7 hervorgeht, können die absoluten Masse hinsichtIich des Wertes ihrer Variationskoeffizienten in 5 Klassen eingeteilt werden. Um den taxonomischen Wert der Parameter dieser Gruppe (A) beurteilen zu können, muss man - unserer Meinung nach - die Variabilität dieser Parameter bei Hunden und bei Wölfen (Wyrost \& $\mathrm{Ku}$ ch a rczyk, 1967 b) vergleichen. Dabei muss man annehmen, dass die Wölfe noch heutzutage in gewissen Gebieten uniforme Populationen darstellen und dass deshalb ihre stabilen Merkmale genotypischer Art sind. Es ist auch bekannt, dass Hunde und Wölfe sehr stark verwandt sind, 
oder auch (R öh r s, 1961; Z e u n e r, 1963), dass die Hunde von den Wölfen abstammen. Unter diesen Umständen behaupten wir, dass die Variabilitätsdifferenzen der stabilen Merkmale bei Wölfen und Hunden hauptsächlich der Domestikation der letzteren zuzuschreiben sind und dass

Tabelle 7 .

Einteilung der Variationskoeffizienten der absoluten Masse (A) in Klassen.

\begin{tabular}{|l|l|l|l|l|}
\hline Klasse & $\begin{array}{c}\text { Merkmals- } \\
\text { variab1littl }\end{array}$ & Wert des $\mathrm{v}$ & $\begin{array}{c}\text { Nerkmals- } \\
\text { anzahl }\end{array}$ & \multicolumn{1}{|c|}{ Merkmalnummern } \\
\hline 1 & sehr schwach & $9,00-13,00$ & 4 & $11,21,12,16$ \\
2 & schwach & $13,01-17,00$ & 1 & 1 \\
3 & mittelmllss1z & $17,01-21,00$ & 5 & $9,3,10,5,19$ \\
4 & stark & $21,01-25,00$ & 8 & $13,17,15,20,14,18,2,6$ \\
5 & sehr stark & $25,01-29,00$ & 3 & $4,8,7$ \\
\hline
\end{tabular}

Tabelle 8 .

Vergleich der Variabilität der absoluten Masse (A) des Hunde- und Wolfsschädels.

\begin{tabular}{|c|c|c|c|c|c|c|c|c|c|c|}
\hline \multirow{2}{*}{ Merimal } & \multicolumn{5}{|c|}{ Can1s lupus L. } & \multicolumn{5}{|c|}{ Canis lupus 2 . famillaris I. } \\
\hline & 1 & 2 & 3 & 4 & 5 & 1 & 2 & 3 & 4 & 5 \\
\hline 11 & 3,24 & - & - & - & - & 9,65 & - & - & - & - \\
\hline 16 & 3,27 & - & - & - & - & 12,86 & - & - & - & - \\
\hline 1 & 3,41 & - & - & - & - & - & 15,66 & - & - & - \\
\hline 18 & 3,42 & - & - & - & - & - & - & - & 22,55 & - \\
\hline 2 & 3,69 & - & - & - & - & - & - & - & 22,70 & - \\
\hline 20 & 3,94 & - & - & - & - & - & - & - & 22,50 & - \\
\hline 5 & 4,00 & - & - & - & - & - & - & 18,87 & - & - \\
\hline 10 & 4,07 & - & - & - & - & - & - & 18,43 & - & - \\
\hline 3 & 4,08 & - & - & - & - & - & - & 18,42 & - & - \\
\hline 6 & 4,45 & - & - & - & - & - & - & - & 24,09 & - \\
\hline 21 & 4,58 & - & - & - & - & 12,12 & - & - & - & - \\
\hline B & 4,81 & - & - & - & - & - & - & - & - & 27,22 \\
\hline 19 & 4,88 & - & - & - & - & - & - & 20,21 & - & - \\
\hline 4 & 5,30 & - & - & - & - & - & - & - & - & 26,36 \\
\hline 12 & - & 5,46 & - & - & - & 12,82 & - & - & - & - \\
\hline 9 & - & 5,52 & - & - & - & - & - & 17,55 & - & - \\
\hline 17 & - & 6,08 & - & - & - & - & - & - & 21,62 & - \\
\hline 15 & - & 6,31 & - & - & - & - & - & - & 22,48 & - \\
\hline 14 & - & - & 8,59 & - & - & - & - & - & 22,64 & - \\
\hline 13 & - & - & 9,45 & - & - & - & - & - & 21,19 & - \\
\hline 7 & - & - & - & - & 14,97 & - & - & - & - & 28,37 \\
\hline
\end{tabular}

diese Merkmale bei Hunden nicht nur genotypischer, sondern auch taxonomischer Natur sind. Je grösser eine Differenz in diesem Sinne zwischen einem stabilen Merkmal des Wolfes und demselben Merkmal des Hundes festgestellt wird, desto grösseren taxonomischen Wert hat dieser Parameter. 
Was die labilen Merkmale betrifft, so kann man sie als von phenotypischer Natur nicht nur beim Wolf, sondern auch beim Hund ansehen. Die Umgebung hat hier bestimmt einen grossen Einfluss auf die starke Variabilität bei beiden Tierarten.

In diesem Sinne können - wie aus Tabelle 8 ersichtlich - zu den (a) genotypischen, aber wenig taxonomischen Parametern die folgenden: $E u$ - Eu (11), Hirnschädelhöhe (16), Länge des Reisszahns (21), und zu den (b) genotypischen und taxonomischen Parametern die folgenden: $O r-P(8), N-P(4)$, Sagekt $-P(6), B-P(2)$, Länge der Backzahn(18), Prämolar- (20) und Molarreihe (19), O-Sagekt (5), grösste Breite des Trigonum occipitale (10), $O-N(3), B-E$ thm (1) gezählt werden; aus Tabelle 3 folgt, dass dies 11 sehr hoch positiv miteinander korrelierte und eindeutig auf die Grösse des Hundeschädels weisenden Masse sind.

Tabelle 9.

Einteilung des Variationskoeffizienten der in Prozenten der Basallänge ausgedrückten absoluten Masse (B) in Klassen.

\begin{tabular}{|c|l|c|c|c|}
\hline Klasse & $\begin{array}{c}\text { Merkmals- } \\
\text { ver1ab1l1tat }\end{array}$ & Wert des $r$ & $\begin{array}{c}\text { Merkmals- } \\
\text { anzahl }\end{array}$ & \multicolumn{1}{|c|}{ Merkmainummern } \\
\hline 1 & sehr sohwach & $4,15-19,15$ & 12 & $\begin{array}{l}106,118,104,120,108,103,105, \\
119,110,121,101,117\end{array}$ \\
2 & schwach & $19,16-34,15$ & 6 & $109,114,115,116,113,111$ \\
3 & mittelmkss18 & $34,16-49,15$ & 1 & 112 \\
4 & stark & $49,16-64,15$ & 0 & 1 \\
5 & sehr staris & $64,16-79,15$ & 107 \\
\hline
\end{tabular}

Aus dem vorher genannten Grunde können zu den zwischen phenound genotypischen Merkmalen gelegenen, die folgenden Schädelmasse gezählt werden: $F t-F t$ (12), $Z y-Z y$ (9), Nasalhöhe (17) und kleinste Gaumenbreite (15). $\mathrm{Zu}$ den phenotypischen Merkmalen gehören der Reihe nach: Höhe des Trigonum frontale (7), Ekt - Ekt (13) und Ent-Ent (14).

Aus diesen Beobachtungen folgt, dass den stabilsten Teil des Hundeund Wolfsschädels der Hirnschädel, den labilsten Teil das Stirndreieck (seine Höhe (7)) und die Stirn in ihrem kleinsten (Ent-Ent) und grösten $(E k t-E k t)$ Breitenmass darstellen.

\section{B. Die absoluten Masse in Prozenten der Basallänge ausgedrïickt}

Wie aus Tabelle 9 ersichtlich, gehören zu den relativ sehr schwach variablen Parametern dieser Gruppe alle Längenmasse, sowie die grösste Breite des Trigonum occipitale und die Nasalhöhe, also alles Masse, die sehr stark mit $B-P$ korreliert sind; zu den mehr oder weniger stabilen 
Parametern der Gruppe B gehören die übrigen Breitenmasse, sowie die Hirnschädelhöhe und die Höhe des Trigonum frontale, also Masse, die mit $B-P$ nicht korreliert sind (Tabelle 3 ).

Wenn wir den Wert der Parameter der Gruppe B nach den gleichen Grundsätzen wie bei den Parametern der Gruppe A beurteilen wollen, so können sie wie folgt eingeteilt werden (Tabelle 10): (a) genotypisch, schwach taxonomisch: Sagekt-P (106), Backzahnreihenlänge (118), $N-P$ (104), Prämolarreihenlänge (120), $O r-P(108), O-N(103), O-$

Tabelle 10.

Vergleich der Variabilität der in Prozenten der Basallänge ausgedrückten absoluten Masse (B) des Hunde- und Wolfsschädels.

\begin{tabular}{|c|c|c|c|c|c|c|c|c|c|c|}
\hline \multirow{2}{*}{ Merkmal } & \multicolumn{5}{|c|}{ Can1s lupus L. } & \multicolumn{5}{|c|}{ Can1s lupus $f$. fam1l1ar1s L. } \\
\hline & 1 & 2 & 3 & 4 & s & 1 & 2 & 3 & 4 & 5 \\
\hline 106 & 2,18 & - & - & - & - & 5,23 & - & - & - & - \\
\hline 108 & 2,22 & - & - & - & - & 9,01 & - & - & - & - \\
\hline 105 & 2,24 & - & - & - & - & 10,59 & - & - & - & - \\
\hline 101 & 2,39 & - & - & - & - & 13,82 & - & - & - & - \\
\hline 103 & 2,74 & - & - & - & - & 9,92 & - & - & - & - \\
\hline 110 & 2,85 & - & - & - & - & 12,43 & - & - & - & - \\
\hline 118 & 2,93 & - & - & - & - & 6,91 & - & - & - & - \\
\hline 104 & 3,05 & - & - & - & - & 7,39 & - & - & - & - \\
\hline 120 & 3,48 & - & - & - & - & 7.54 & - & - & - & - \\
\hline 116 & 3,60 & - & - & - & - & - & 23,72 & - & - & - \\
\hline 109 & 3,82 & - & - & - & - & - & 19,37 & - & - & - \\
\hline 111 & 3,93 & - & - & - & - & - & 26,23 & - & - & - \\
\hline 121 & - & 4,19 & - & - & - & 13,30 & - & - & - & - \\
\hline 115 & - & 4,77 & - & - & - & - & 20,93 & - & - & - \\
\hline 117 & - & 4,86 & - & - & - & 14,75 & - & - & - & - \\
\hline 119 & - & 4,99 & - & - & - & 10,69 & - & - & - & - \\
\hline 112 & - & 5,49 & - & - & - & - & - & 38,83 & - & - \\
\hline 114 & - & - & 7,27 & - & - & - & 19,87 & - & - & - \\
\hline 113 & - & - & 8,09 & - & - & - & 23,88 & - & - & - \\
\hline 107 & - & - & - & - & 15,12 & - & - & - & - & 78,07 \\
\hline
\end{tabular}

Sagekt (105), grösste Breite des Trigonum occipitale (110), B - Ethm (101), also 9 Parameter, deren Zähler sehr hoch positiv mit dem Nenner $B-P$ korreliert sind (Tabelle 3), (b) genotypisch und taxonomisch: $E u-E u$ (111), Hirnschädelhöhe (116), $Z y-Z y$ (109).

Die übriggebliebenen Parameter sind entweder phenotypischer Natur [Höhe des Trigonum frontale (107), Ekt-Ekt (113), Ent-Ent (114)] oder liegen zwischen den pheno- und genotypischen [Länge des Reiss- 
zahns (121), kleinste Gaumenbreite (115), Nasalhöhe (117), Länge der Molarreihe (119), Ft - Ft (112)].

\section{Die Quotienten-Indizes}

Die Variabilität der Indizes dieser Gruppe ist, wie aus Tabelle 11 ersichtlich, sehr unterschieden. Ihr taxonomischer Wert, ähnlich wie vorher beurteilt, sieht folgendermassen aus (Tabelle 12): (a) genotypische, schwach taxonomische Parameter: Backzahn-Index (210), Breiten-Höhen-

\section{Tabelle 11.}

Einteilung des Variationskoeffizienten der Quotienten-Indizes (C) in Klassen.

\begin{tabular}{|c|c|c|c|c|}
\hline Klasse & $\begin{array}{l}\text { We rknals- } \\
\text { variabilitat }\end{array}$ & Wert des $v$ & $\begin{array}{l}\text { Merknals- } \\
\text { snzahl }\end{array}$ & Merkmalnummern \\
\hline 1 & sehr schwach & $3,41-12,41$ & 6 & $210,209,206,211,208,207$ \\
\hline 2 & schirach & $12,42-21,41$ & 3 & $2 C 5,201,204$ \\
\hline 3 & m1t telmass $1 \mathrm{~g}$ & $21,42-30,41$ & 1 & 203 \\
\hline 4 & stark & $30,42-39,41$ & 0 & \\
\hline 5 & sehr staric & $39,42-48,41$ & 1 & 202 \\
\hline
\end{tabular}

Tabelle 12.

Vergleich der Variabilität der Quotienten-Indizes (C) des Hunde- und Wolfsschädels.

\begin{tabular}{|l|c|c|c|c|c|c|c|c|c|c|}
\hline \multirow{2}{*}{ Merkanal } & \multicolumn{9}{|c|}{ Can1s lupus L. } & \multicolumn{5}{|c|}{ Can1s lupus 1. fam111ar1s L. } \\
\cline { 2 - 9 } & 1 & 2 & 3 & 4 & 5 & 1 & 2 & 3 & 4 & 5 \\
\hline 210 & 1,15 & - & - & - & - & 4,23 & - & - & - & - \\
205 & 3,25 & - & - & - & - & - & 12,60 & - & - & - \\
207 & 3,32 & - & - & - & - & 11,46 & - & - & - & - \\
209 & 3,83 & - & - & - & - & 6,29 & - & - & - & - \\
211 & 4,00 & - & - & - & - & 9,43 & - & - & - & - \\
201 & 4,30 & - & - & - & - & - & 13,58 & - & - & - \\
206 & - & 5,35 & - & - & - & 7,02 & - & - & - & - \\
208 & - & 5,45 & - & - & - & 10,72 & - & - & - & - \\
204 & - & 5,47 & - & - & - & - & 18,48 & - & - & - \\
203 & - & - & 8,09 & - & - & - & - & 23,88 & - & - \\
202 & - & - & - & - & 18,02 & - & - & - & - & 47,59 \\
& & - & & & & & & & & - \\
\hline
\end{tabular}

-Index (209), Reisszahn-Index (211), (b) genotypische und taxonomische Parameter: Längen-Breiten-Index (201), Stirnlage-Index (205), Höhen-Längen-Index (207).

Die übrigen Parameter der Gruppe C sind entweder phenotypisch oder dazwischen gelegen. $\mathrm{Zu}$ den ersten gehören: Stirndreieck-Index (202) und Stirnbreiten-Index (203), zu den letzteren: Breitendifferenz-Index (206), Ausprägungs-Index (208) und Höhendifferenz-Index (204). 
Was die Relation der Parameter aller Gruppen zur Grösse der Tiere, die sehr gut in unserem Material durch $B-E t h m$ (Wyrost \& $\mathrm{Ku}$ c h a r c z y k, 1967a) repräsentiert ist, betrifft, so können sie zwei Klassen zugezählt werden (Tabellen 3,4): 1) Von der Grösse des Hundes in hohem Grad abhängige (Korrelation mit Merkmal Nr. 1 über $90 \%$, also hoch positiv), 2) Von der Grösse des Hundes wenig oder überhaupt nicht abhängige. Zur ersten Klasse gehören von den Parametern der Gruppe A: $O-N(3), B-P(2), N-P(4), O-$ Sagekt (5), Sagekt $-P(6), O r-P$ (8), grösste Breite des Trigonum occipitale (10), also ausser dem letztgenannten alles Längenmasse, und von den Indizes - nur der zur Gruppe C gehörende Längen-Breiten-Index des Hirnschädels (201). Zur zweiten Klasse gehören die 9 übrigen Höhen- und Breitenmasse (Nr. 17, 14, 9, $13,16,15,11,7,12)$ und die 8 übrigen Quotienten-Indizes (Nr. 205, 208, $202,204,207,203,206,209)$. Eine den Wert von $90 \%$ überschreitende Korrelation zwischen Parametern der Gruppe B und B-Ethm wurde nicht festgestellt. Sehr nahe dieser Grenze liegt der Parameter Nr. 111 (Eu$E u$ ), welcher mit $B-E$ thm eine negative Korrelation in der Höhe von $88,1 \%$ aufweist.

Was die Variabilität betrifft, so scheinen - wie aus einem Vergleich der Mittelwerte der Variationskoeffizienten der Parameter der Gruppen A und C folgt - die Hundeschädel sich mehr in der Grösse $\left(\bar{V}_{A}=19,9 \%\right)$ als in der Gestalt $\left(V_{\mathrm{C}}=15,0 \%\right)$ zu unterscheiden.

Wie aus der dargestellten Analysis hervorgeht, kann der sehr in Gestalt und Grösse unterschiedliche Hundeschädel, von Rasse und Geschlecht abgesehen, am günstigsten und bequemsten durch die folgenden 17 - von den 52 insgesamt betrachteten - Parametern charakterisiert werden (Tabellen $8,10,12$ ):

1) Parameter der Gruppe A: $O r-P(8), N-P(4)$, Sagekt $-P(6)$, $B-P(2)$, Länge der Backzahnreihe (18), Länge der Prämolarreihe (20), Länge der Molarreihe (19), O-Sagekt (5), Grösste Breite des Trigonum occipitale (10), $O-N$ (13) und B-Ethm (1);

2) Parameter der Gruppe B: $E u-E u$ (111) und Hirnschädelhöhe (116), eventuell noch $Z y-Z y$ (109);

3) Parameter der Gruppe C: Längen-Breiten-Index des Hirnschädels (201), Stirnlage-Index (205) und eventuell auch Höhen-Längen-Index des Hirnschädels (207).

Der taxonomische Wert dieser Parameter für eine Segregation des kraniologischen Materials des Hundes ist gross. Diese Parameter sind beim Hunde wahrscheinlich genotypischer Natur und in dieser Beziehung unterscheiden sich die verschieden gestalteten Hundeschädel am meisten. Auf dieser Grundlage kann - nach unserer Ansicht - den Parametern 
dieser Gruppe ein erstklassiger Wert für die richtige Beurteilung des Hundeschädels zugesprochen werden.

Die übrigen Parameter sind entweder genotypisch aber schwach taxonomisch für viele Hunderassen (Nr. 11, 16, 21, 101, 103-106, 108, 110, $118,120,209,210,211$ ) oder dazwischen gelegen (zweitklassige Parameter: $9,12,15,17,112,115,117,119,121,204,206,208)$ oder phenotypisch

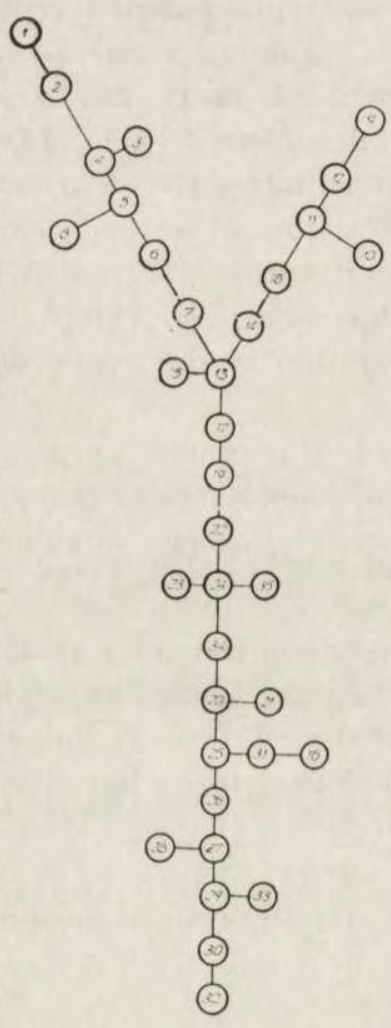

Abb. 19

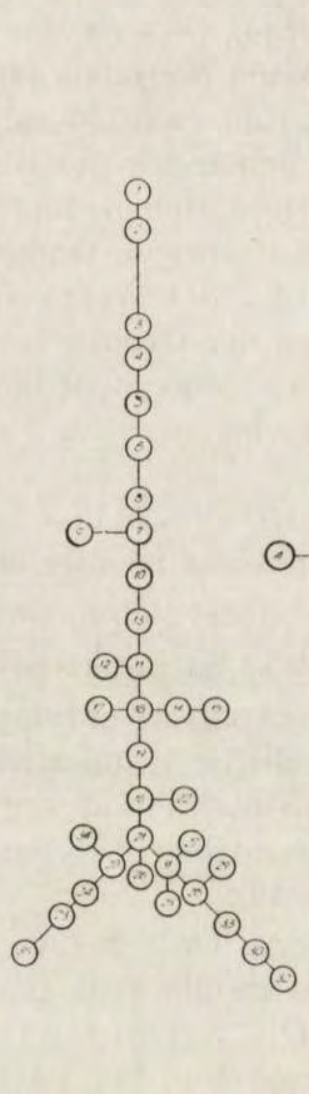

Abb. 20.

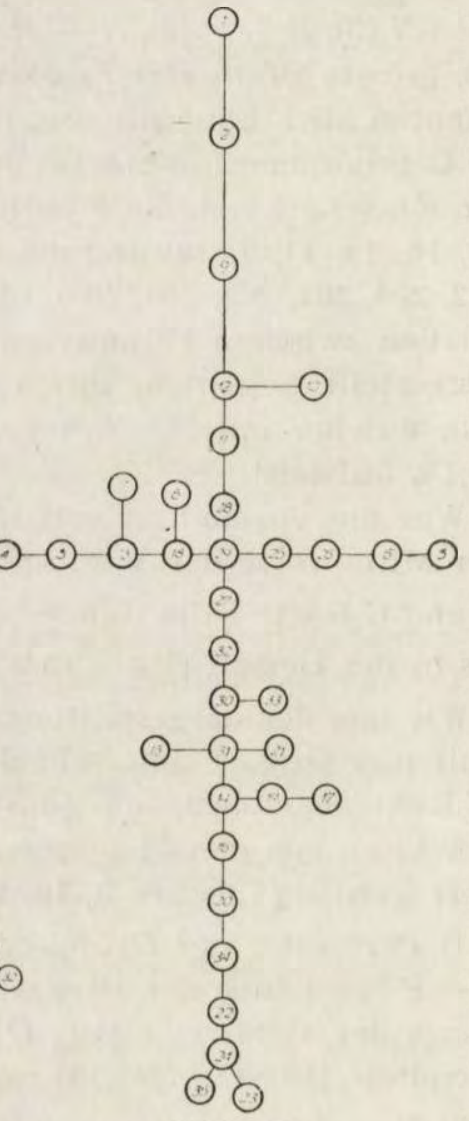

Abb. 21.

Abb. 19. Auf 17 absoluten Massen $(1-17)$ basierter Graph.

Abb. 20. Auf 8 erstklassigen absoluten Massen $(1-6,8,10)$ basierter Graph. Abb. 21. Auf 16 in Prozenten der Basallänge ausgedrückten absoluten Massen (101, 103-117) basierter Graph.

(drittklassige Parameter: 7, 13, 14, 107, 113, 114, 202, 203). Ihr Gebrauch in Untersuchungen ist für eine vollere Charakteristik der Grösse, besonders aber der Gestalt des Hundeschädels sehr oft begründet.

Über die Segregation unseres in Grösse und Gestalt unterschiedlich gestalteten Materials auf Grund der untersuchten Parameter geben die 
6 angeführten Graphen Auskunft. Der erste (Abb. 19) wurde anhand von 17 (Nr. 1-17), der zweite (Abb. 20) anhand von 8 (Nr. 1-6, 8, 10) Parametern der Gruppe A, der dritte (Abb. 21) anhand von 16 (Nr. 101, 103117), der vierte (Abb. 22) anhand von 3 (Nr. 109, 111, 116) Parametern der Gruppe B, der fünfte (Abb. 23) anhand von 9 (Nr. 201-209) und der sechste (Abb. 24) anhand von 3 (Nr. 201, 205, 207) Parametern der Gruppe C aufgestellt. Der erste, dritte und fünfte Graph wurde demnach an-

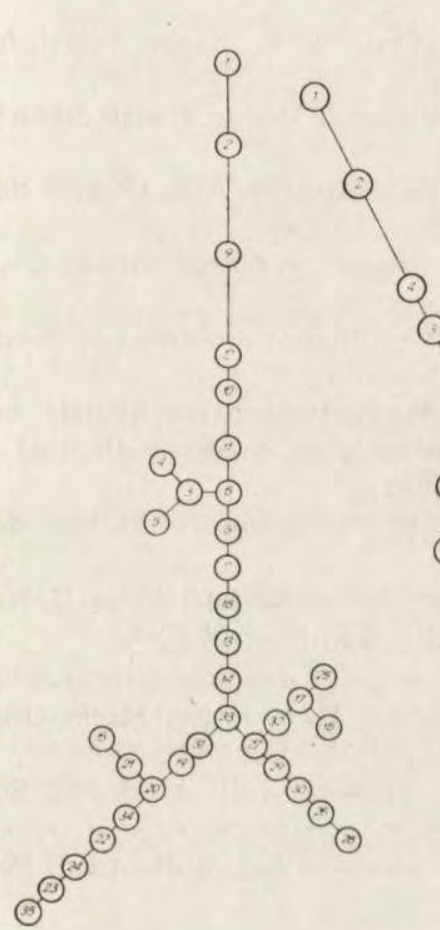

Abb. 22 .

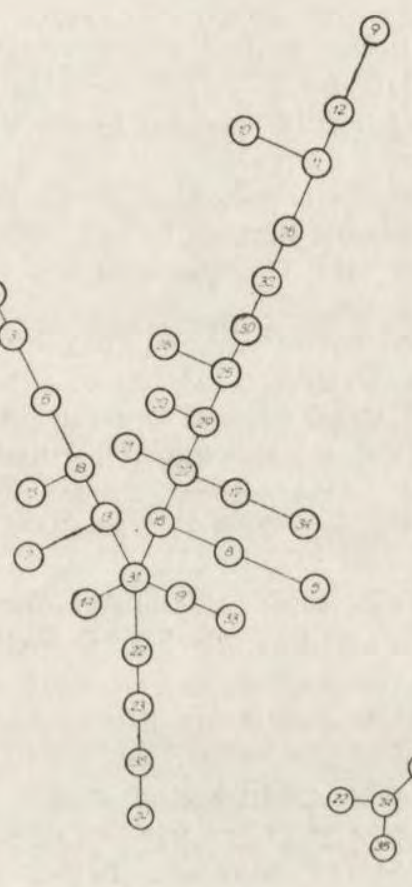

Abb, 23,

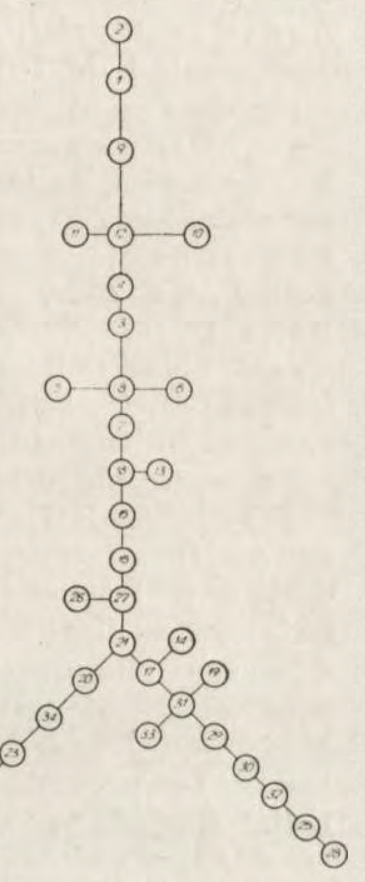

Abb. 24.

Abb. 22. Auf 3 erstklassigen in Prozenten der Basallänge ausgedrückten absoluten Massen $(109,111,116)$ basierter Graph.

Abb. 23. Auf 9 Quotienten-Indizes (201-209) basierter Graph.

Abb. 24. Auf 3 erstklassigen Quotienten-Indizes (201, 205, 207) basierter Graph.

hand von allen, der zweite, vierte und sechste Graph anhand von erstklassigen Parametern entsprechend der Gruppen A, B und C aufgebaut.

Die Gestalt der Graphen und die Konfiguration der Schädel sagen aus, dass ein Aufstellen der Graphen anhand von erstklassigen Parametern nicht nur weniger Rechenarbeit verursacht, sondern auch ein ähnliches oder sogar mehr wirklichkeitstreues Bild gibt als alle Parameter zusammen. Diese Tatsache bekräftigt unsere Ansicht, dass die Parameter, die 
von uns als erstklassig, also genotypisch und taxonomisch erklärt wur-den, in Wirklichkeit die Hauptrolle in der Hundeschädeldiagnostik spielen. Die anderen Parameter können hier nur eine ergänzende Rolle haben.

\section{SCHRIFTTUM}

1. Bibikova V. I., 1953: Fauna rannetripolskogo poselenja Luka-Vrobleveckaja. Mat. Issled. po Archeol. SSSR, 38: 411-458.

2. Bohlken H., 1961: Haustiere und zoologische Systematik. Ztschr. Tierzücht. Züchtungsbiol., 76, 1: 107-113.

3. Brinkmann A., 1921: Canidenstudien I-III. Vidensk, Meddr. Dansk Naturh. Foren., 72. Kjobenhavn.

4. Brin kmann A., 1924: Canidenstudien V-VI. Bergens Mus. Arb. 1923/24. Naturvidensk. Raekke, 7: 1-57. Bergen.

5. B o golubskij S. N., 1928: Ob analogičnyh priznakah v čerepe sobak. Russ. Zool. Ž., 8, 3: 7-32.

6. Da hr E., 1937: Studien über Hunde aus primitiven Steinzeitkulturen in Nordeuropa. Lunds Univ. Arskrift NF, Avd. 2, 32, 4. Lund.

7. Duerst J. U., 1930: Vergleichende Untersuchungsmethoden am Skelett bei Säugern. (In Abderhalden E.: Handbuch der biologischen Arbeitsmethoden) 1, 7: 125-530. Urban und Schwarzenberg. Berlin-Wien.

8. Georgi W., 1937: Rassen- und funktionelle Merkmale am Unterkiefer des Hundes. Diss. Leipzig.

9. Götze \& Dornheim, 1926: Messungen und variationsstatistische Untersuchungen an Haushundschädeln. Ztschr. Tierzücht. Züchtungsbiol., 5.

10. Kl a t t B., 1913: Ubber den Einfluss der Gesamtgrösse auf das Schädelbild nebst Bemerkungen über die Vorgeschichte der Haustiere. Arch. Entw. Mech. Org., 36: 387-471. Leipzig.

11. M a r chlewski T., 1926: Craniological studies on dogs. Bull. Acad. Pol. Sci. Lett.

12. Marchlewski T., 1930: Craniology of the domestic dog. Bull. Acad. Pol. Sci. Lett., Cl. B.

13. Perka 1 J., 1953: Taksonomia wrocławska. Przegl. Antropol., 19: 82-96.

14. Perkal J., 1958-1963: Matematyka dla przyrodników i rolników, 1-3. Państw. Wyd. Nauk.: 1-254, 1-314, 1-358. Warszawa.

15. Reményi K., 1954: Profilpoligon, egy ùj koponyamérési eljárás. Bilógiai Közlemények, 2, 1-2: 93-109.

16. Röhrs M., 1961: Biologische Anschauungen über Begriff und Wesen der Domestikation. Ztschr. Tierzücht. Züchtungsbiol., 76, 1: 7-23.

17. Schäme R., 1922: Die Grundformen des Haushundschädels. Jb. Jagdkde., 4.

18. Studer T., 1901: Die prähistorischen Hunde in ihrer Beziehung zu den gegenwärtig lebenden Rassen. Abh. Schweiz. Palaeont. Ges., 28: 1-137.

19. W a gner K., 1929: Rezente Hunderassen. Eine osteologische Untersuchung. Skr. Norske Vidensk-Akad., Mat.-Naturv. K1., 9: 1-157. Oslo.

20. Wyrost P. \& K ucharczyk J., 1967a: Versuch der Bestimmung der Widerristhöhe der Hundes mittels der inneren Hirnhöhlenlänge. Acta theriol., 12, 9: $105-110$. 
21. Wyrost P. \& K u charczyk J., 1967b: Uber einige Eigenschaften gewisser Schädelsmerkmale der osteuropäischen Wolfes. Zool. pol. (Im Druck).

22. Z e un er F., 1963: A history of domesticated animals. Hutchinson. 1-560. London.

Eingang des Ms. 17. März 1967.

Lehrstuhl für Tieranatomie der Hochschule für Agrikultur in Wrocław, I.ehrstuhl für Statistik der Universität Wrocław,

Wrocław 12, Kożuchowska $1-3$.

Piotr WYROST \& Jerzy KUCHARCZYK

\section{ANALIZA PRZYDATNOSCI NIEKTORYCH PARANETRÓW CZASZKI PSA DO JEJ MORFOLOGICZNEJ OCENY}

\section{Streszczenie}

Badania nad przydatnością niektórych parametrów czaszki psa (Canis lupus f. familiaris Linnaeus, 1758) do jej morfologicznej oceny przeprowadzono na niezbyt licznym, ale znacznie w kształcie i wielkości zróżnicowanym materiale złożonym z czaszek (calvaria) 35 dorosłych psów (8 samic i 27 samców) 19 różnych ras o wysokości w kłębie od 20 do $80 \mathrm{~cm}$ (Tab. 1). W badaniach tych wzięto pod uwagę 52 parametry: A. 21 pomiarów absolutnych wyrażonych $\mathrm{w} m m(1-21)$, B. 20 pomiarów absolutnych wyrażonych w odsetkach $B-P(101-121)$ i C. 11 wskaźników ilorazowych (201-211). Morfologiczną i taksonomiczną wartość tych cech ustalano w pracy przez badanie zachodzących między tymi parametrami korelacji (Tab, 3 i 4), analizowanie współczynników zmienności tych cech (Tab. 2, 5, 6), porównywanie otrzymanych informacji z takimi samymi właściwościami takich samych parametrów czaszki wilka (Canis lupus Linna e us, 1758) (Tab. 8, 10, 12), grupowanie materiału według cech w szeregi rozdzielcze (Tab. $7,9,11$ ) oraz przez analizowanie sporządzonych z tych parametrów, według niżej zamieszczonego wzoru, tablic odległości Czekanowskiego i wykreślonych na ich podstawie dendrytów (Ryc. 19-24):

$$
\mathrm{d}=\sqrt{\frac{1}{\mathrm{n}} \sum_{1}^{\mathrm{n}}\left(\mathrm{x}_{\mathrm{i}}-\mathrm{y}_{\mathrm{i}}\right)^{2}}
$$

gdzie $d$ wyraża odległość miẹdzy osobnikniem $x$ i $y$, a $n$ ilość branych pod uwage cech. Dla każdego z wyżej wymienionych parametrów obliczono też w pracy zakres jego zmienności $(Z)$, rozstęp $(R)$, średnią arytmetyczną $(\bar{x})$ i odchylenie standardowe $(S)$. Wszystkie potrzebne do badań obliczenia wykonano w Katedrze Metod Numerycznych Uniwersytetu Wroclawskiego na maszynie cyfrowej „Elliott 803”.

W wyniku przeprowadzonych badań doszliśmy do następujących wniosków końcowych:

1. Z trzech branych w pracy pod uwagę grup cech (A, B i C), do parametrów genotypowych taksonomicznych u psa najprawdopodobniej należą: z pomiarów absolutnych (A): $O r-P, N-P$, Sagekt $-P, B-P$, długość szeregu zębów policzkowych, przedtrzonowych i trzonowych, $O$-Sagekt, największa szerokość trójkąta potylicznego, $O-N$ i $B-E t h m$, z pomiarów wyrażonych w odsetkach $B-P(\mathrm{~B}): E u-E u$, wy- 
sokość puszki mózgowej i ewentualnie $Z y-Z y$, a $z$ wskaźników ilorazowych (C): wsk. wydłużenia puszki mózgowej, wsk. położenia czoła Brinkmanna i wsk. wysokościowo-długościowy puszki mózgowej. Tymi to cechami różne w kształcie i wielkości czaszki psów różnią się między sobą najistotniej. Cechy pozostałe, to albo $\mathrm{z}$ genotypowych cechy mało dla czaszki psa taksonomiczne $(11,16,21,101,103$ $106,108,110,118,120,209-211)$ albo cechy fenotypowe $(7,13,14,107,113,114,202$, 203) albo cechy wartości pośredniej między geno- a fenotypowymi $(9,12,15,17,112$, $115,117,119,121,204,206,208$ ).

2. Z wysokością psa w kłębie (W) skorelowana jest bardzo wysoko $(r=+96,1 \%)$ wewn. długości puszki mózgowej (1) - jeden z pięciu stosunkowo bardzo stabilnych pomiarów czaszki psa, zwłaszcza wśród zwierząt tej samej wielkości. Wnosząc z zachodzącej korelacji między tym pomiarem a parametrami pozostałymi, do cech w znacznym stopniu rzutujących na wielkość psa należą: z pomiarów absolutnych (A); oprócz pomiaru nr 1: $O-N, B-P, N-P, O-$ Sagekt, Sagekt-P, Or-P i największa szerokość trójkąta potylicznego, a z cech typu B i C: wsk. wydlużenia puszki mózgowej oraz ewentualnie wyrażona w odsetkach $B-P$ szerokość puszki mózgowej - należą tu zatem parametry bardzo wysoko i z wyjątkiem parametru ostatniego dodatnio ze sobą skorelowane.

3. Pod względem zmienności, czaszki psów wydają siẹ być bardziej zróżnicowane w wielkości niż w kształcie. Najbardziej stałą ich częścią jest puszka mózgowa, a najbardziej zmienna - trójkąt czołowy (jego wysokość) i czoło w swym największym. (Ekt-Ekt) i najmniejszym poprzecznym wymiarze (Ent-Ent). Z trzech podstawowych pomiarów puszki mózgowej $(E u-E u$, wysokość i $B-E t h m)$, najbardziej zmiennym i niewątpliwie w głównej mierze warunkującym ksztalt jamy czaszki i pośrednio formę zawartego w niej mózgowia, jest B-Ethm - parametr, który jako jedyny $\mathrm{z}$ wyżej wymienionych trzech jest bardzo wysoko i dodatnio skorelowany $\mathrm{z}$ wielkością psa.

Nakład $840+150$ egz. Obj. ark. wyd. 11. Maszynopis otrzymano 21.III.67 r. Podpisano do druku 5.VII.1967 r. Druk ukończono w lipcu 1967 r. Papier druk. sat. kl. III 80 g. Format B5.

Cena $35 \mathrm{zl}$

Białostockie Zakłady Graficzne. Zam. 1621* M-2 